\title{
Highly Sensitive Real-Time Isotopic Quantification of Water by ATR-FTIR
}

Cei B. Provis-Evans ${ }^{1,2}$, Elliot H. E. Farrar ${ }^{1}$, Matthew N. Grayson ${ }^{1}$, Ruth L. Webster ${ }^{1 *}$, Alfred K. Hill ${ }^{3 *}$

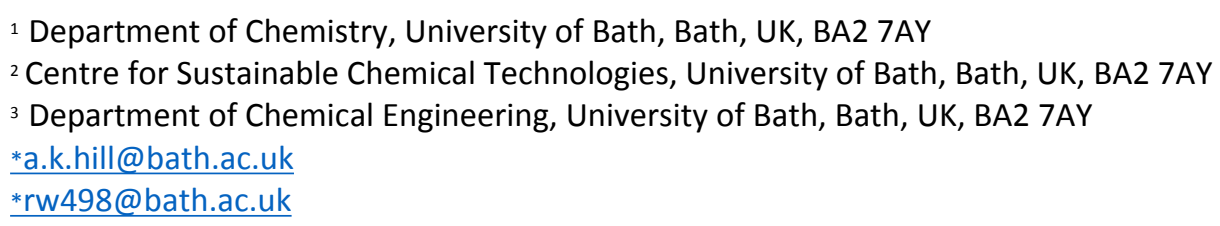

\section{Table of Contents}

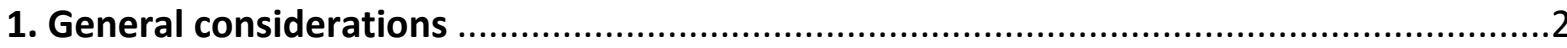

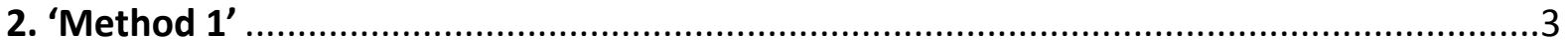

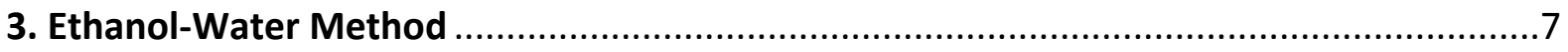

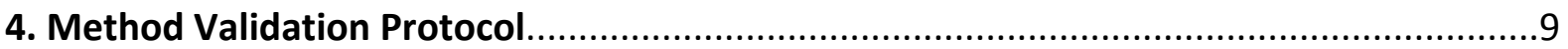

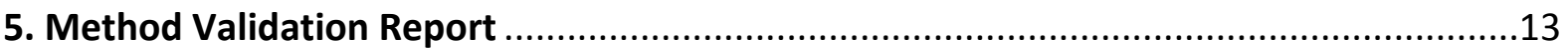

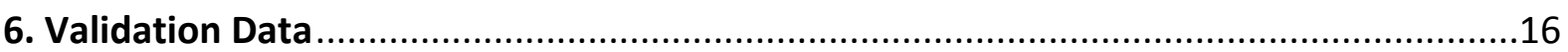

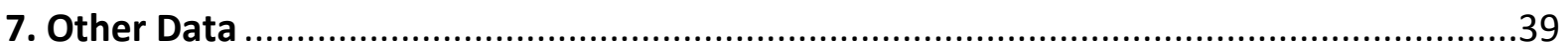

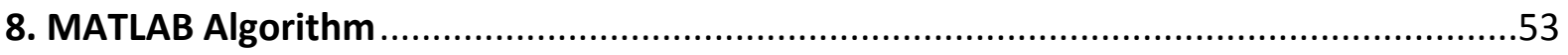

9. Computational methods and additional considerations ..........................................56

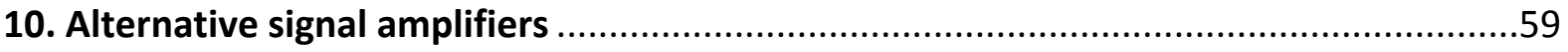

11. Energies and molecular geometries of all computed structures ..............................60

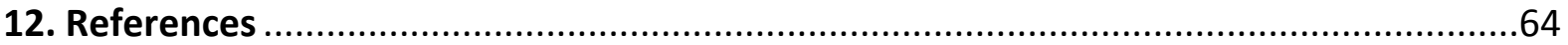




\section{General considerations}

\section{Instrumentation}

All ATR-FTIR performed using a Mettler Toledo ReactIR 15 instrument; ${ }^{1}$ a 'DiComp' diamond ATR cell connected to a liquid N2 cooled mercury cadmium telluride infrared detector by means of a $6 \mathrm{~mm} \times 1.5 \mathrm{~m}$ silver halide optical fibre. Temperature and $\mathrm{pH}$ measured using a Hanna Instruments HI 83141 pH meter/temperature probe, calibrated as per instructions using $\mathrm{pH} 10$ (borate) and $\mathrm{pH} 4$ (phthalate) buffer solutions from Fisher traceable to NIST pH standards.

\section{Chemicals}

Deuterium depleted or enriched water, along with the other chemicals used were purchased from Sigma Aldrich, Alfa Aesar or Fisher. DI water was obtained from a reverse osmosis plant within the chemistry building.

\section{Equipment}

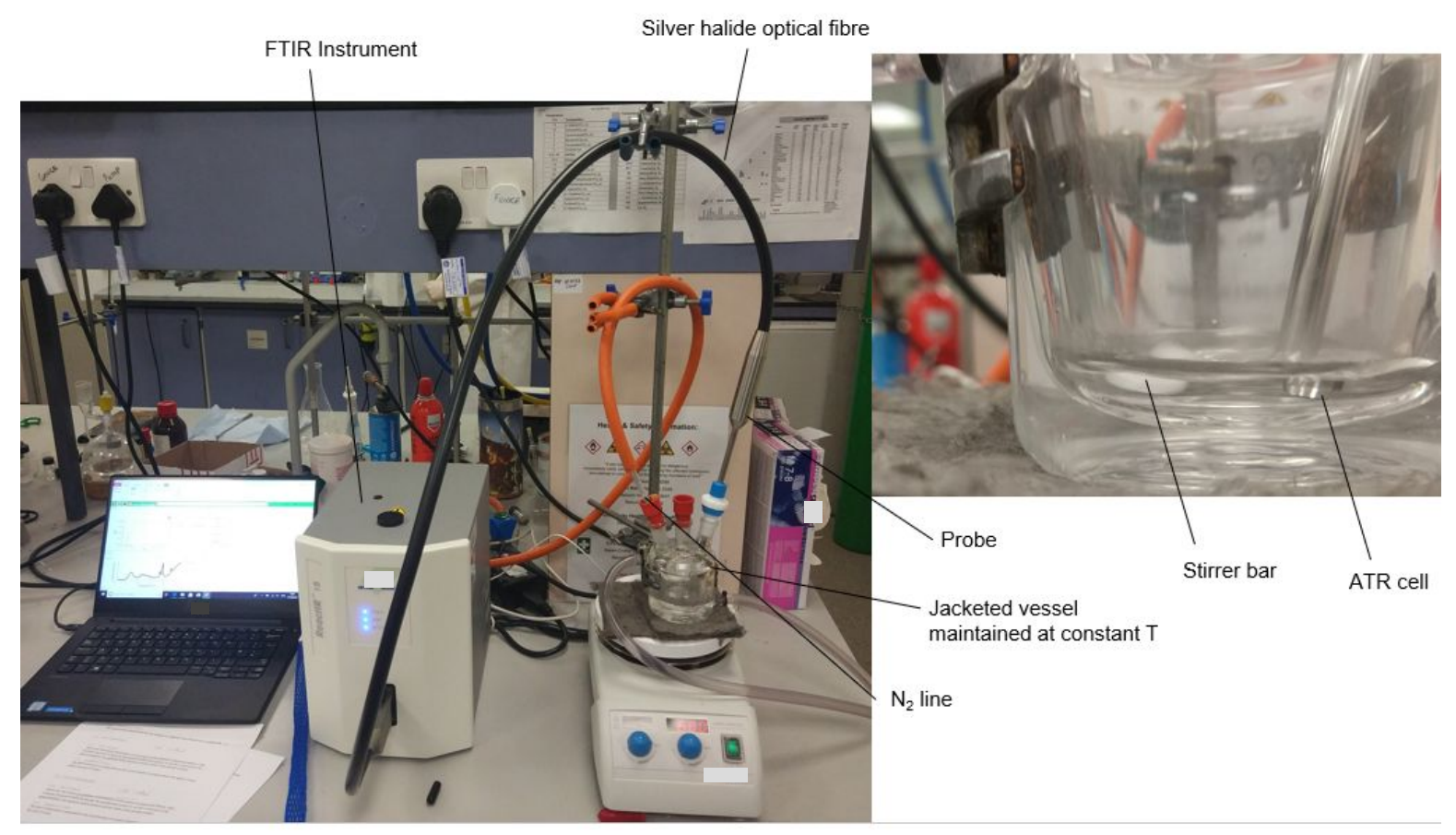

Figure S1. Experimental arrangement for FTIR experiments using the ReactIR. 


\section{2. 'Method 1'}

\section{Scope}

This method is intended to be used in confirming the suitability of the ReactIR instrument for use in quantifying the concentration of deuterium atoms in water by the spectroscopic detection of singly deuterated water (HDO).

\section{Test Method Description}

This method uses an ATR-FTIR probe which is wholly immersed in the analyte and attached to a Mettler Toledo ReactIR 15 instrument which can continually collect FTIR spectra and log these to accurately quantify HDO and therefore deuterium atom concentration over time.

\section{Equipment and Supplies}

Mettler Toledo ReactIR 15 instrument

DiComp (diamond) ATR cell, connected via silver halide optical fibre cable Volumetric Flasks $1 \mathrm{ml}$ and $200 \mu \mathrm{l}$ autopipettes Round bottomed flask / jacketed vessel with minimum of two necks Magnetic stirrer plate and stirrer bar Method for sealing the system (suba-seals)

Cryostat

\section{Reagents and Standards}

Deuterium depleted water (<1ppm D atom)

Deuterium oxide (100 D atom \%)* 
*If this method were to be used for absolute quantification of deuterium in unknown samples, a deuterium standards traceable to Vienna Standard Mean Ocean Water (VSMOW) would be used here.

\section{Procedure}

\section{A. Preparation of Calibration Solution}

Pipette $500 \mu \mathrm{l}$ of deuterium oxide ( $\geq 99.95 \% \mathrm{D}$ atom) into a $5 \mathrm{ml}$ volumetric flask. Add to this $4000 \mu$ l of deuterium depleted water $(<1 \mathrm{ppm} D)$ to give water with a nominal $12.4504 \mathrm{D}$ atom \% (124504 ppm) deuterium atom concentration, or 249008 ppm of $\mathrm{HDO}$ in $\mathrm{H}_{2} \mathrm{O}$.

\section{B. Operational Parameters}

The probe is placed in a vessel with at least two openings, maintained at a constant $15^{\circ} \mathrm{C}$ using an external cryostat and with a magnetic stirrer placed in such as way as to not interfere with the IR probe. The system is sealed during determinations under air.

The ReactIR instrument is set-up per the user instructions, taking the background of the system with the dry probe as close to its test position as possible. Parameters for IR spectra determinations are as follows:

Resolution: Normal (Every 8 wavenumbers)

No. of Scans: $\quad 256$

Scanning Frequency: 1 scan/minute

Averaging: $\quad 4$ determinations averaged; 1 data point/ 4 minutes

Scan Range: $\quad 1900-700 \mathrm{~cm}^{-1}$ 


\section{Calibration and System Suitability}

Add $5000 \mu$ of deionised water (with natural deuterium abundance) to the vessel and place the IR probe so that the end is immersed in the water. Begin the data collection, with one spectrum being taken every 1 minutes using 256 scans. Allow the system to equilibrate (at least 1 hour), then add the calibration standard in steps as specified below. For each, allow one spectrum to be recorded while the sample becomes homogenous, then record four spectra which once averaged will be used as the calibration data. Once these have been obtained, carry out the next addition as per table below, again allowing one homogenisation and four calibration spectra to be recorded. Transfer the data obtained to the iCQuant software package and interpret the spectra using the following parameters:

Functions: No correction method

Integration: Area to two-point baseline,

Peak: $\quad 1465$ and $1400 \mathrm{~cm}^{-1}$

Baseline: $\quad 1535$ and $1340 \mathrm{~cm}^{-1}$

Deionised water plus four sequential additions of $100 \mu \mathrm{l}$ standard affords a 5-point calibration.

The deuterium atom concentration of the calibration solution therefore varies according to the table S1 below:

Table S1. Calibration data for deuterium in water measured by ATR FTIR.

\begin{tabular}{r|cccc} 
NO. & $\begin{array}{c}\text { VOLUME DI } \\
\text { H2O }\end{array}$ & $\begin{array}{c}\text { TOTAL STD } \\
\text { ADDED }\end{array}$ & $\begin{array}{c}\text { TOTAL } \\
\text { VOL }\end{array}$ & D CONC (PPM) \\
\hline $\mathbf{1}$ & 5000 & 0 & 5000 & 156 \\
$\mathbf{2}$ & 5000 & 100 & 5100 & 2594 \\
$\mathbf{3}$ & 5000 & 200 & 5200 & 4939 \\
$\mathbf{4}$ & 5000 & 300 & 5300 & 7195
\end{tabular}




\begin{tabular}{l|llll}
5 & 5000 & 400 & 5400 & 9367
\end{tabular}

The resulting model can then be transferred back into the data collection software and used to quantify the unknown deuterium content of samples.

After the final standard addition, the system should be allowed to continue collecting data for 6 further determinations to evaluate the system variability. The system suitability requirements are as follows:

Calibration correlation coefficient: $\quad \geq 0.995$

SD of six further determinations: $\quad \leq 50 \mathrm{ppm}$

\section{Determination}

The sample should be gently pipetted into the vessel, immersing the head of the IR probe. This should be allowed to equilibrate until the signal stabilises and then quantified according to the quantitative model produced from the calibration data. Determinations can be continuous, so this method can be used to track changing concentrations of deuterium in a flow of water. In order to minimise the variability of the system, determinations should be averaged over four spectra.

When introducing a fresh sample, the previous sample should be pipetted out and the vessel rinsed with bench acetone then dried under a flow of nitrogen or dry air. The next sample can then be introduced, being careful to ensure that no bubbles form under the probe which may interfere with the determination. 


\section{Ethanol-Water Method}

\section{Spectroscopic parameters}

$\begin{array}{ll}\text { Resolution: } & \text { Every } 8 \text { wavenumbers } \\ \text { No. of Scans/Spectrum: } & 256 \\ \text { Frequency: } & 1 \text { spectrum/minute } \\ \text { Averaging: } & \text { None } \\ \text { Scan Range: } & 1900-700 \mathrm{~cm}^{-1} \\ \text { Integration: } & \text { Area to two-point baseline, } \\ \text { Peak: } & 955 \text { and } 910 \mathrm{~cm}^{-1} \\ \text { Baseline: } & 960 \text { and } 890 \mathrm{~cm}^{-1}\end{array}$

\section{Calibration parameters}

A standard water sample with 1992 ppm deuterium atom concentration was prepared following the approach given in method 1 above. $5000 \mu \mathrm{l}$ of ethanol (with natural deuterium abundance) was added to the vessel and place the IR probe so that the end is immersed. The system was equilibrated for at least one hour, then the calibration standard was added in steps as specified below in Table 1 . One spectrum was recorded while the sample became homogenous and calibration data was the average of four subsequent spectra.

Table S2. Calibration parameters for ethanol - water system.

$\begin{array}{ccccc}\text { No. } & \text { volume Ethanol } & \text { Total std added } & \text { total vol } & \text { D conc (ppm) } \\ 1 & 5000 & 0 & 5000 & 156 \\ 2 & 5000 & 10 & 5010 & 159 \\ 3 & 5000 & 20 & 5020 & 163 \\ 4 & 5000 & 30 & 5030 & 167 \\ 5 & 5000 & 40 & 5040 & 170\end{array}$


The system suitability requirements for the calibration correlation coefficient: was $\geq$ 0.995 and the standard deviation of six further determinations was $\leq 50 \mathrm{ppm}$. Determinations were made according to Method 1 above. 


\section{Method Validation Protocol}

\section{Purpose}

The purpose of this validation protocol is to describe the experiments to be performed and the associated acceptance criteria for the validation of Method 1 and the ReactIR instrument:

\section{Test Method Description}

The test method under validation is 'Method 1'. This method attempts to show the suitability of the ReactIR instrument for determining the amount of deuterium in $\mathrm{H}_{2} \mathrm{O}$ by evaluating the area under the H-O-D scissoring bend vibrational mode in IR spectra obtained using an ATRFTIR probe immersed in the test solution.

\section{Necessary Equipment}

ReactIR 15 Instrument

ATR-FTIR probe, connected to instrument via silver halide optical fibre

Schlenk flasks

Volumetric flasks

Autopipettes

Jacketed Flask

4. Method

Method 1 


\section{Procedure}

\subsection{Linearity/Accuracy}

\subsubsection{Test Procedure}

Standard solutions will be prepared at six concentrations over the range of the technique (156 - 9367 ppm D atom). Three individually prepared replicates at each concentration level will be analysed. 10 readings (40 minutes) of data in deionised water will also be collected to evaluate the standard deviation of the noise.

\subsubsection{Acceptance Criteria}

The correlation coefficient for six concentration levels will be $\geq 0.999$ over the stated range of the method. All samples should fall within two standard deviations of the target concentration.

\subsection{Range}

\subsubsection{Test Procedure}

The data obtained during the linearity /accuracy study will be used to assess the range of the method. Precision data for this assessment is from the three replicate samples analysed at each level.

\subsubsection{Acceptance Criteria}

The acceptable range will be defined as the concentration interval over which linearity and accuracy are observed per the above criteria, and in addition, that yields a precision of $\leq 3 \% \mathrm{RSD}$ 


\subsection{Precision - Repeatability}

\subsubsection{Test Procedure}

One sample containing the target level (4939 ppm) of analyte will be prepared. Six replicates will be made from this sample solution as per the procedure outlined in the method.

\subsubsection{Acceptance Criteria}

RSD should be $\leq 2 \%$ over the six replicate determinations

\subsection{Intermediate Precision}

\subsubsection{Test Procedure}

Intermediate precision will be demonstrated by two analysts on different days with separately obtained calibration curves. The data for the first analyst can be taken from the linearity/accuracy experiment, and the second analyst will prepare and quantify the central two samples of the accuracy experiment.

\subsubsection{Acceptance Criteria}

The assay results obtained by the two analysts on different days should have an RSD $\leq$ $4 \%^{2}$ 


\subsection{Limit of Detection}

\subsubsection{Test Procedure}

Determine the lowest detectable concentration of the analyte by the limit of blank (LOB) method. After calibration of the system, take at least 60 determinations of a sample of D-depleted water used to prepare the standard $(5 \mathrm{ml})$ and determine the SD of these determinations. ${ }^{3}$

$$
\begin{aligned}
& \mathrm{LOB}=\text { mean }_{\text {blank }}+1.645\left(\mathrm{SD}_{\text {blank }}\right) \\
& \mathrm{LOD}=\mathrm{LoB}+1.645\left(\mathrm{SD}_{\text {low concentration sample }}\right)
\end{aligned}
$$

\subsection{Limit of Quantification}

\subsubsection{Test Procedure}

Determine the lowest quantifiable concentration of the analyte as defined by the concentration where accuracy and precision of 60 determinations fulfil the acceptance criteria outlined elsewhere in this document. LOQ cannot be lower than LOD. ${ }^{3}$

\subsection{Robustness}

\subsubsection{Test Procedure}

The system will be calibrated in the normal way, then a variety of parameters will be changed and a $4939 \mathrm{ppm}$ sample will be quantified as per the method. The parameters to be changed are temperature $\left( \pm 5^{\circ} \mathrm{C}\right), \mathrm{pH}(2-10)$, ionic strength (addition of $1 \% \mathrm{w} / \mathrm{v}$ $\mathrm{NaCl}), \pm \mathrm{N}_{2}$. At least three determinations should be made under each of the changed parameters. 


\subsubsection{Acceptance Criteria}

Sample assay concentration should continue to fall within $2.5 \%$ of the target concentration.

\section{Method Validation Report}

\section{Method Validation}

In order to demonstrate that Method 1 as outlined above is suitable for use to quantify deuterium concentration and to determine the limitations of the method, a validation protocol was written, and the experiments detailed within it were carried out (see ESI for protocol and data). Here follows a brief description of each experiment and the conclusions obtained. See Table 1 for a summary of the data. Acceptance criteria were set with reference to ICH advice for the validation of pharmaceutical analytical methods, ${ }^{4}$ and to more specific guidance from Shabir. ${ }^{5}$ Validations of other FTIR quantification methods were also inspected for comparison, and acceptance criteria adjusted accordingly. ${ }^{2}$

\section{Linearity/Accuracy}

Standard solutions were prepared at six concentration levels within the calibrated range of the technique:

Level 1: 156 ppm D

Level 2: 1387 ppm D

Level 3: 2594 ppm D

Level 4: 4939 ppm D

Level 5: 7195 ppm D

Level 6: 9367 ppm D

Three replicate measurements were carried out at each concentration level. The vessel was cleaned and dried between each replicate determination. 
All samples fell within two SDs of the target concentrations, with the SD taken from 10 repeat determinations on deionised (DI) water taken earlier in the run, and the $R^{2}$ of the six determinations was 0.9999 against acceptance criteria of $\geq 0.9990$.

\section{Precision-Repeatability}

One sample at the midpoint of the calibration (4939 ppm D) was prepared and six replicate determinations were conducted on it.

The relative standard deviation (RSD) of the six determinations was $1.6 \%$ while the acceptance criteria was $\leq 2.0 \%$

\section{Intermediate Precision}

Two analysts on different days quantified the central two levels ( 3 and 4 ) of the Linearity experiment with three replicate determinations, and the results compared.

The RSD of the two analysts combined data fell within the acceptance criteria of $4.0 \%$ in both cases.

\section{Limit of Detection (LOD)}

The lowest detectable concentration of deuterium was established using the limit of blank (LOB) method, using 60 determinations of deuterium depleted water over a period of 240 minutes, in addition to 60 determinations of $\mathrm{DI}$ water as a low deuterium concentration sample. ${ }^{3}$ The equations used are shown below:

$\mathrm{LOB}=$ mean $_{\text {blank }}+1.645\left(\mathrm{SD}_{\text {blank }}\right)$

$\mathrm{LOD}=\mathrm{LOB}+1.645\left(\mathrm{SD}_{\text {low }}\right.$ concentration sample $)$

The SD of 60 repeated determinations was $43 \mathrm{ppm} \mathrm{D,} \mathrm{which} \mathrm{in} \mathrm{combination} \mathrm{with} \mathrm{the} \mathrm{LOB} \mathrm{data}$ gave a value for the LOD of $137 \mathrm{ppm}$ D. 


\section{Limit of Quantification (LOQ)}

The lowest quantifiable concentration of deuterium is defined as the concentration where the accuracy and precision of 60 determinations fulfil the acceptance criteria outlined for the precision and accuracy experiments. ${ }^{3}$

The 60 determinations carried out on $\mathrm{DI}$ water with the natural abundance of deuterium comply with these criteria, and therefore the LOQ was established as $156 \mathrm{ppm} \mathrm{D.}$

\section{Robustness}

The system was calibrated under normal conditions as per Method 1, and the $4939 \mathrm{ppm} D$ standard (level 4) was quantified with parameters changed to ascertain if the method is robust to such changes. The parameters which were changed were temperature $\left( \pm 5^{\circ} \mathrm{C}\right), \mathrm{pH}$ (2 - 10), ionic strength $(1-6 \% \mathrm{w} / \mathrm{v} \mathrm{NaCl})$ and with and without an atmosphere of dry nitrogen. The acceptance criteria were that the concentration of each determination should fall within $2.5 \%$ of the target concentration, with a minimum of three determinations conducted for each varied parameter.

These experiments ascertained that the system was not robust to changes in temperature of $2{ }^{\circ} \mathrm{C}$ or more, or to an increase in ionic strength of $6 \% \mathrm{w} / \mathrm{v}$ against the conditions under which it was calibrated. However, the system was robust to $\mathrm{pH}$ changes from 2 - 10, an increase in ionic strength of $1 \% \mathrm{w} / \mathrm{v}$ and against exposure to ambient atmospheric conditions between the calibration and the testing.

Table S3. Summary Table of Method Validation

\begin{tabular}{|c|c|c|c|c|}
\hline EXPERIMENT & CRITERIA & $\begin{array}{l}\text { ACCEPTANCE } \\
\text { CRITERIA }\end{array}$ & DATA OBTAINED & PASS/FAIL \\
\hline ACCURACY/LINEARITY & $\begin{array}{l}\text { Distance from } \\
\text { target }\end{array}$ & $<2 \mathrm{SD}$ & $\begin{array}{l}\text { All samples fell } \\
\text { within } 2 \mathrm{SD}\end{array}$ & Pass \\
\hline & $R^{2}$ & $\geq 0.9990$ & 0.9999 & Pass \\
\hline PRECISION-REPEATABILITY & RSD & $\leq 2.0 \%$ & $1.6 \%$ & Pass \\
\hline
\end{tabular}




\begin{tabular}{|c|c|c|c|c|}
\hline \multirow[t]{2}{*}{ INTERMEDIATE PRECISION } & RSD & $\leq 4.0 \%$ & Level $3=3.5 \%$ & Pass \\
\hline & & & Level $4=3.0 \%$ & Pass \\
\hline LOD & - & - & 137 ppm D & $\mathrm{N} / \mathrm{A}$ \\
\hline LOQ & - & - & 156 ppm D & $\mathrm{N} / \mathrm{A}$ \\
\hline \multirow[t]{7}{*}{ ROBUSTNESS } & Temperature & $<2.5 \%$ deviation & $\pm 2{ }^{\circ} \mathrm{C},>2.5 \%$ & Fail \\
\hline & & & $\pm 5^{\circ} \mathrm{C},>2.5 \%$ & Fail \\
\hline & $\mathrm{pH}$ & $<2.5 \%$ deviation & $\mathrm{pH} 2^{+}<2.5 \%$ & Pass \\
\hline & & & $\mathrm{pH} 10^{\ddagger}<2.5 \%$ & Pass \\
\hline & Ionic strength & $<2.5 \%$ deviation & $1 \%,<2.5 \%$ & Pass \\
\hline & & & $6 \%,>2.5 \%$ & Fail \\
\hline & Remove $\mathrm{N}_{2}$ & $<2.5 \%$ deviation & $<2.5 \%$ & Pass \\
\hline INSTRUMENTAL SD & $\begin{array}{l}60 \text { repeat } \\
\text { determinations }\end{array}$ & - & 43 ppm D & $\mathrm{N} / \mathrm{A}$ \\
\hline
\end{tabular}

\section{Validation Data}

Table S4. Linearity/Accuracy: Noise evaluation

\begin{tabular}{rr|rll} 
NO & \multicolumn{1}{|r}{ READING } & AVG/4 & \multicolumn{1}{l}{$\boldsymbol{\delta}$ D (\%o) } \\
\hline $\mathbf{1}$ & 77.32 & & \\
$\mathbf{2}$ & 216.96 & & \\
$\mathbf{3}$ & 113.84 & & \\
$\mathbf{4}$ & 216.43 & 156.1375 & 2.74198 \\
$\mathbf{5}$ & 216.9 & & \\
$\mathbf{6}$ & 102.05 & & \\
$\mathbf{7}$ & 106.83 & & \\
$\mathbf{8}$ & 244.12 & 167.475 & 75.58315 \\
$\mathbf{9}$ & 287.27 & & \\
$\mathbf{1 0}$ & 164.97 & & \\
$\mathbf{1 1}$ & 53.51 & & \\
$\mathbf{1 2}$ & 291.08 & 199.2075 & 279.4405 \\
$\mathbf{1 3}$ & 97.18 & & \\
\hline $\mathbf{1 4}$ & 165.68 & & \\
$\mathbf{1 5}$ & 129.45 & & \\
$\mathbf{1 6}$ & 13.07 & 101.345 & -349.277 \\
$\mathbf{1 7}$ & 49.59 & & \\
$\mathbf{1 8}$ & 99.68 & & \\
$\mathbf{1 9}$ & 263.51 & & \\
$\mathbf{2 0}$ & 78.61 & 122.8475 & -211.161 \\
$\mathbf{2 1}$ & 73.76 & & \\
$\mathbf{2 2}$ & 107.42 & & \\
$\mathbf{2 3}$ & 10.54 & & \\
$\mathbf{2 4}$ & 165.13 & 89.2125 & -427.091 \\
& & & \\
\hline
\end{tabular}




\begin{tabular}{|c|c|c|c|}
\hline 25 & 141.15 & & \\
\hline 26 & 250.6 & & \\
\hline 27 & 58.72 & & \\
\hline 28 & 139.81 & 147.57 & -52.3526 \\
\hline 29 & 255.71 & & \\
\hline 30 & 210.32 & & \\
\hline 31 & 252.31 & & \\
\hline 32 & 138.77 & 214.2775 & 376.0202 \\
\hline 33 & 208.93 & & \\
\hline 34 & 121.19 & & \\
\hline 35 & 86.84 & & \\
\hline 36 & 196.34 & 153.325 & -15.3402 \\
\hline 37 & 138.85 & & \\
\hline 38 & 112.9 & & \\
\hline 39 & 222.64 & & \\
\hline 40 & 230.17 & 176.14 & 131.217 \\
\hline SD & 76.58632 & 39.88892 & 256.2041 \\
\hline
\end{tabular}

Table S5. Linearity/Accuracy Data

LEVEL REPLICATE STD $(\mu \mathrm{L})$ TARGET READING AVERAGE RESIDUAL CONC

\begin{tabular}{r|rrrrrr}
$\mathbf{1}$ & 1 & 0 & 156.00 & 202 & & \\
$\mathbf{3}$ & 2 & 0 & 156.00 & 115 & & \\
& 3 & 0 & 156.00 & 111 & 143 & -13 \\
& 1 & 50 & 1387.17 & 1321 & & \\
& 2 & 50 & 1387.17 & 1341 & & \\
& 3 & 50 & 1387.17 & 1420 & 1361 & -26 \\
& 1 & 100 & 2594.20 & 2694 & & \\
& 2 & 100 & 2594.20 & 2603 & & \\
& 3 & 100 & 2594.20 & 2616 & 2638 & 44 \\
& 1 & 200 & 4938.62 & 4879 & & \\
& 2 & 200 & 4938.62 & 4938 & & \\
& 3 & 200 & 4938.62 & 4934 & 4917 & -22 \\
& 1 & 300 & 7194.57 & 7141 & & \\
& 2 & 300 & 7194.57 & 7273 & & \\
& 3 & 300 & 7194.57 & 7223 & 7212 & 18 \\
& 1 & 400 & 9366.97 & 9393 & & \\
& 2 & 400 & 9366.97 & 9330 & & \\
& 3 & 400 & 9366.97 & 9271 & 9331 & -36
\end{tabular}




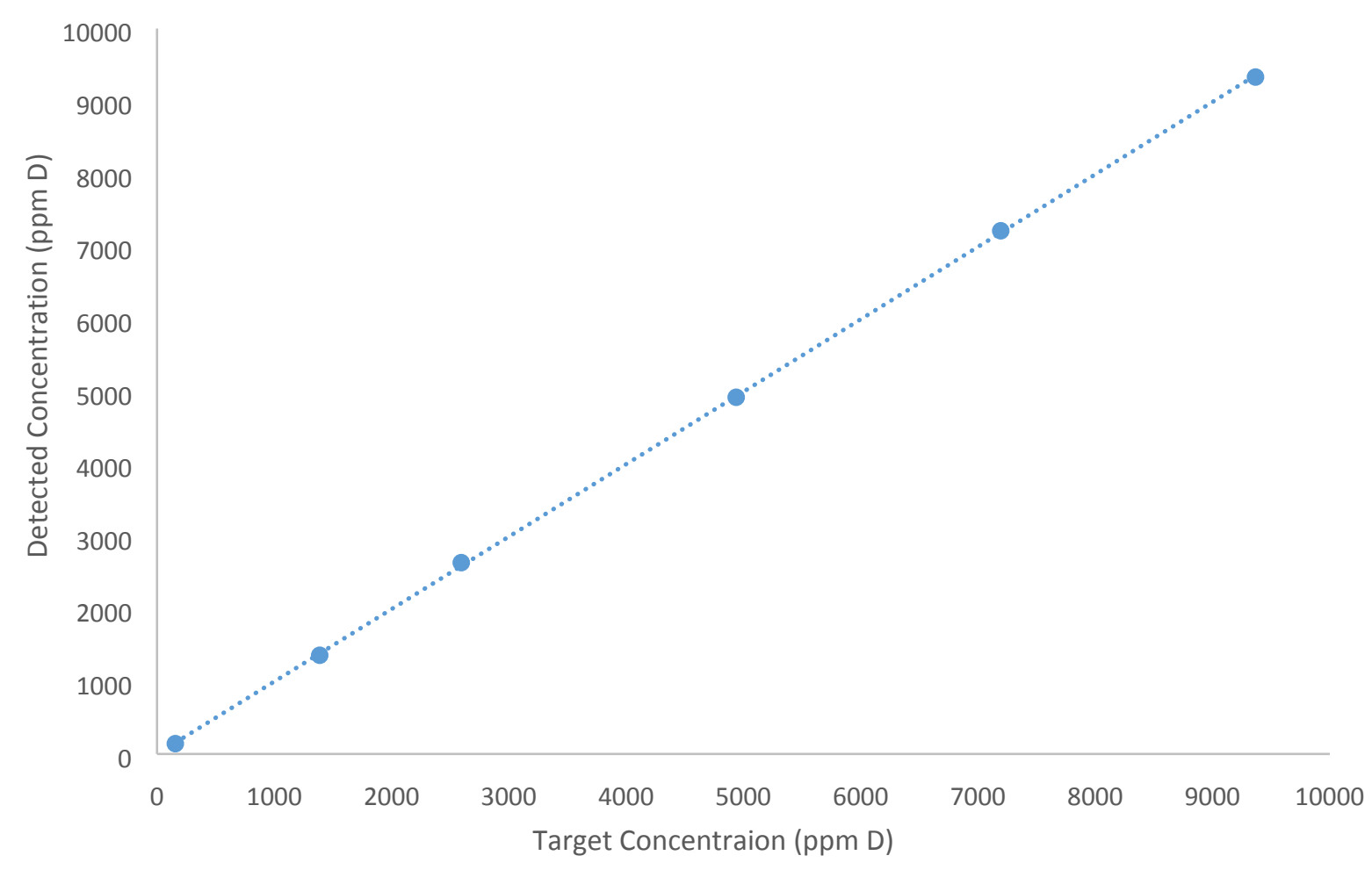

Figure S2. Calibration data showing the linearity and accuracy by $\mathrm{R}^{2}$ method.

Table S6. Precision Data

\begin{tabular}{|c|c|c|c|c|}
\hline REPLICATE & TARGET & $\begin{array}{l}\text { RAW } \\
\text { DATA }\end{array}$ & AVERAGES & $\begin{array}{l}\text { \% CHANGE } \\
\text { FROM TARGET }\end{array}$ \\
\hline \multirow[t]{4}{*}{1} & 4939 & 4,937 & & \\
\hline & 4939 & 4,864 & & \\
\hline & 4939 & 4,911 & & \\
\hline & 4939 & 4,891 & 4901 & -0.76652 \\
\hline \multirow[t]{4}{*}{2} & 4939 & 4,824 & & \\
\hline & 4939 & 4,894 & & \\
\hline & 4939 & 4,722 & & \\
\hline & 4939 & 4,791 & 4808 & -2.65014 \\
\hline \multirow[t]{4}{*}{3} & 4939 & 4,743 & & \\
\hline & 4939 & 4,837 & & \\
\hline & 4939 & 4,826 & & \\
\hline & 4939 & 4,886 & 4823 & -2.33994 \\
\hline \multirow[t]{4}{*}{4} & 4939 & 5,004 & & \\
\hline & 4939 & 5,065 & & \\
\hline & 4939 & 4,912 & & \\
\hline & 4939 & 4,913 & 4973 & 0.703932 \\
\hline \multirow[t]{4}{*}{5} & 4939 & 4,874 & & \\
\hline & 4939 & 4,946 & & \\
\hline & 4939 & 4,857 & & \\
\hline & 4939 & 4,942 & 4905 & -0.68613 \\
\hline
\end{tabular}




\begin{tabular}{|l|rrrr|}
\hline 6 & 4939 & 4,793 & \\
& 4939 & 4,739 & & \\
& 4939 & 4,712 & & -3.60284 \\
& 4939 & 4,799 & 4761 & \\
& & & & \\
SD & & & 78.05 & \\
AVERAGE & & & 4861.73 & \\
RSD & & & $1.61 \%$
\end{tabular}

Table S7. Intermediate Precision

\begin{tabular}{c|rrrrrrrr} 
LEVEL & $\begin{array}{l}\text { ANALYST - } \\
\text { REPLICATE }\end{array}$ & $\begin{array}{l}\text { STD ADDED } \\
(\boldsymbol{\mu} \text { L })\end{array}$ & $\begin{array}{l}\text { TARGET } \\
\text { CONC }\end{array}$ & READING & AVERAGE & SD & AVG & RSD \\
\hline 3 & CW-1 & 100 & 2594 & 2469 & & & & \\
\hline & CW-2 & 100 & 2594 & 2568 & & & & \\
& CW-3 & 100 & 2594 & 2468 & 2502 & & & \\
& CPE-1 & 100 & 2594 & 2694 & & & & \\
& CPE-2 & 100 & 2594 & 2603 & & & & \\
& CPE-3 & 100 & 2594 & 2616 & 2570 & 88.71 & 2570 & 3.45 \\
& CW-1 & 200 & 4939 & 4685 & & & & \\
& CW-2 & 200 & 4939 & 5141 & & & & \\
& CW-3 & 200 & 4939 & 4927 & 4918 & & & \\
& CPE-1 & 200 & 4939 & 4879 & & & & \\
& CPE-2 & 200 & 4939 & 4938 & & & & \\
& CPE-3 & 200 & 4939 & 4934 & 4918 & 145.78 & 4918 & 2.96
\end{tabular}

Table S8. LOD-Limit of Blank

Blank (D-depleted water)

\begin{tabular}{|c|c|c|c|}
\hline $\begin{array}{l}\text { DETERMINATION } \\
\text { NO }\end{array}$ & $\begin{array}{l}\text { D ATOM } \\
\text { PPM }\end{array}$ & AVERAGE/4 & $\delta \mathrm{D}(\%)$ \\
\hline 1 & 2.35 & & \\
\hline
\end{tabular}




\begin{tabular}{|c|c|c|c|}
\hline & -9.78 & & \\
\hline & -35.07 & & \\
\hline & 109.16 & 16.665 & -892.979 \\
\hline \multirow[t]{4}{*}{2} & 158.76 & & \\
\hline & -153.7 & & \\
\hline & 38.84 & & \\
\hline & -45.8 & -0.475 & -1003.05 \\
\hline \multirow[t]{4}{*}{3} & 133.52 & & \\
\hline & 68.8 & & \\
\hline & -24.05 & & \\
\hline & -79.8 & 24.6175 & -841.908 \\
\hline \multirow[t]{4}{*}{4} & -31.13 & & \\
\hline & 19.84 & & \\
\hline & -6.45 & & \\
\hline & 35.52 & 4.445 & -971.455 \\
\hline \multirow[t]{4}{*}{5} & -33.1 & & \\
\hline & 215.02 & & \\
\hline & -95.04 & & \\
\hline & 53.95 & 35.2075 & -773.897 \\
\hline \multirow[t]{4}{*}{6} & 89.68 & & \\
\hline & -130.95 & & \\
\hline & -26.72 & & \\
\hline & 18.92 & -12.2675 & -1078.78 \\
\hline \multirow[t]{4}{*}{7} & -30.92 & & \\
\hline & 72.56 & & \\
\hline & 151.73 & & \\
\hline & -44.35 & 37.255 & -760.748 \\
\hline \multirow[t]{4}{*}{8} & -15.74 & & \\
\hline & 128.24 & & \\
\hline & 101.15 & & \\
\hline & 54.45 & 67.025 & -569.551 \\
\hline \multirow[t]{4}{*}{9} & -64.3 & & \\
\hline & -60.79 & & \\
\hline & 35.81 & & \\
\hline & 13.21 & -19.0175 & -1122.12 \\
\hline \multirow[t]{4}{*}{10} & 36.12 & & \\
\hline & -74.34 & & \\
\hline & 50.97 & & \\
\hline & 65.58 & 19.5825 & -874.243 \\
\hline \multirow[t]{4}{*}{11} & 49.86 & & \\
\hline & -61.51 & & \\
\hline & -4.52 & & \\
\hline & -64.72 & -20.2225 & -1129.86 \\
\hline \multirow[t]{3}{*}{12} & 9.25 & & \\
\hline & 154.24 & & \\
\hline & -1.37 & & \\
\hline
\end{tabular}




\begin{tabular}{|c|c|c|c|}
\hline & -116.76 & 11.34 & -927.176 \\
\hline \multirow[t]{4}{*}{13} & -1.75 & & \\
\hline & -55 & & \\
\hline & 111.37 & & \\
\hline & 64.82 & 29.86 & -808.24 \\
\hline \multirow[t]{4}{*}{14} & -94.27 & & \\
\hline & -5.48 & & \\
\hline & -95.37 & & \\
\hline & 80.17 & -28.7375 & -1184.54 \\
\hline \multirow{4}{*}{15} & 110.79 & & \\
\hline & -12.14 & & \\
\hline & 49.65 & & \\
\hline & 5.93 & 38.5575 & -752.383 \\
\hline \multirow[t]{4}{*}{16} & 74.39 & & \\
\hline & -24.83 & & \\
\hline & -2.38 & & \\
\hline & 136.67 & 45.9625 & -704.825 \\
\hline \multirow[t]{4}{*}{17} & -6.6 & & \\
\hline & 29.12 & & \\
\hline & 0.75 & & \\
\hline & 22.57 & 11.46 & -926.406 \\
\hline \multirow[t]{4}{*}{18} & 87.05 & & \\
\hline & -1.92 & & \\
\hline & -56.56 & & \\
\hline & -126.39 & -24.455 & -1157.04 \\
\hline \multirow[t]{4}{*}{19} & -26.98 & & \\
\hline & 65.97 & & \\
\hline & 20.49 & & \\
\hline & 57.24 & 29.18 & -812.607 \\
\hline \multirow[t]{4}{*}{20} & -111.34 & & \\
\hline & 122.16 & & \\
\hline & -105.67 & & \\
\hline & -156.6 & -62.8625 & -1403.66 \\
\hline \multirow[t]{4}{*}{21} & -25.77 & & \\
\hline & -23.42 & & \\
\hline & -10.96 & & \\
\hline & 58.54 & -0.4025 & -1002.58 \\
\hline \multirow[t]{4}{*}{22} & 189.64 & & \\
\hline & -161.81 & & \\
\hline & 13.66 & & \\
\hline & 84.15 & 31.41 & -798.285 \\
\hline \multirow[t]{4}{*}{23} & 125.45 & & \\
\hline & -23.5 & & \\
\hline & -26.82 & & \\
\hline & -2.25 & 18.22 & -882.993 \\
\hline 24 & 85.99 & & \\
\hline
\end{tabular}




\begin{tabular}{|c|c|c|c|}
\hline & -32.27 & & \\
\hline & -85.35 & & \\
\hline & -103.38 & -33.7525 & -1216.74 \\
\hline \multirow[t]{4}{*}{25} & -88.04 & & \\
\hline & -49.12 & & \\
\hline & 46.03 & & \\
\hline & 22.83 & -17.075 & -1109.65 \\
\hline \multirow[t]{4}{*}{26} & -95.16 & & \\
\hline & 111.23 & & \\
\hline & -34.56 & & \\
\hline & -31.63 & -12.53 & -1080.46 \\
\hline \multirow[t]{4}{*}{27} & -148.41 & & \\
\hline & -100.79 & & \\
\hline & 221.5 & & \\
\hline & 87.94 & 15.06 & -903.286 \\
\hline \multirow[t]{4}{*}{28} & -26.04 & & \\
\hline & -104.84 & & \\
\hline & 69.42 & & \\
\hline & 89.79 & 7.0825 & -954.517 \\
\hline \multirow[t]{4}{*}{29} & -67.9 & & \\
\hline & 26.39 & & \\
\hline & -73.35 & & \\
\hline & -47.93 & -40.6975 & -1261.34 \\
\hline \multirow[t]{4}{*}{30} & -112.23 & & \\
\hline & 69.67 & & \\
\hline & -76.2 & & \\
\hline & -117.25 & -59.0025 & -1378.88 \\
\hline \multirow[t]{4}{*}{31} & 3.67 & & \\
\hline & -173.99 & & \\
\hline & 10.65 & & \\
\hline & -126.29 & -71.49 & -1459.06 \\
\hline \multirow[t]{4}{*}{32} & -165.78 & & \\
\hline & -124.31 & & \\
\hline & -32.23 & & \\
\hline & -254.01 & -144.0825 & -1925.13 \\
\hline \multirow[t]{4}{*}{33} & 52.19 & & \\
\hline & -81.6 & & \\
\hline & -174.56 & & \\
\hline & -67.63 & -67.9 & -1436.01 \\
\hline \multirow[t]{4}{*}{34} & -104.67 & & \\
\hline & -54.47 & & \\
\hline & -54.36 & & \\
\hline & -96.38 & -77.47 & -1497.46 \\
\hline \multirow[t]{3}{*}{35} & -73.59 & & \\
\hline & -71.51 & & \\
\hline & -86.82 & & \\
\hline
\end{tabular}




\begin{tabular}{|c|c|c|c|}
\hline & -9.95 & -60.4675 & -1388.29 \\
\hline \multirow[t]{4}{*}{36} & 17.66 & & \\
\hline & 92.35 & & \\
\hline & -85.81 & & \\
\hline & -269 & -61.2 & -1392.99 \\
\hline \multirow[t]{4}{*}{37} & 18.16 & & \\
\hline & -113.15 & & \\
\hline & 49.56 & & \\
\hline & -61.96 & -26.8475 & -1172.4 \\
\hline \multirow[t]{4}{*}{38} & -13.45 & & \\
\hline & -122.86 & & \\
\hline & -112.39 & & \\
\hline & -2.61 & -62.8275 & -1403.44 \\
\hline \multirow[t]{4}{*}{39} & -46.09 & & \\
\hline & 1.83 & & \\
\hline & 15.68 & & \\
\hline & -99.48 & -32.015 & -1205.59 \\
\hline \multirow[t]{4}{*}{40} & -106.65 & & \\
\hline & 6.16 & & \\
\hline & 24.99 & & \\
\hline & 50.32 & -6.295 & -1040.42 \\
\hline \multirow[t]{4}{*}{41} & 119.21 & & \\
\hline & -62.35 & & \\
\hline & -71.62 & & \\
\hline & 55.15 & 10.0975 & -935.155 \\
\hline \multirow[t]{4}{*}{42} & 42.85 & & \\
\hline & 98.5 & & \\
\hline & -98.72 & & \\
\hline & 109.22 & 37.9625 & -756.204 \\
\hline \multirow[t]{4}{*}{43} & -78.3 & & \\
\hline & -102.85 & & \\
\hline & -28.99 & & \\
\hline & 99.62 & -27.63 & -1177.43 \\
\hline \multirow[t]{4}{*}{44} & -91.13 & & \\
\hline & 56.32 & & \\
\hline & 7.74 & & \\
\hline & -54.52 & -20.3975 & -1130.99 \\
\hline \multirow[t]{4}{*}{45} & -151.34 & & \\
\hline & 47.78 & & \\
\hline & 26.59 & & \\
\hline & 49.79 & -6.795 & -1043.64 \\
\hline \multirow[t]{4}{*}{46} & -90.22 & & \\
\hline & -80.14 & & \\
\hline & -9.62 & & \\
\hline & 55.16 & -31.205 & -1200.39 \\
\hline 47 & -6.3 & & \\
\hline
\end{tabular}




\begin{tabular}{|c|c|c|c|}
\hline & -215.5 & & \\
\hline & -206.84 & & \\
\hline & 17.25 & -102.8475 & -1660.4 \\
\hline \multirow[t]{4}{*}{48} & -221.3 & & \\
\hline & -134.87 & & \\
\hline & -11.93 & & \\
\hline & -61.87 & -107.4925 & -1690.22 \\
\hline \multirow[t]{4}{*}{49} & -163.35 & & \\
\hline & 33.54 & & \\
\hline & -131.63 & & \\
\hline & -61.09 & -80.6325 & -1517.76 \\
\hline \multirow[t]{4}{*}{50} & -39.2 & & \\
\hline & -25.73 & & \\
\hline & -36.41 & & \\
\hline & -147.93 & -62.3175 & -1400.16 \\
\hline \multirow[t]{4}{*}{51} & -23.17 & & \\
\hline & 9.71 & & \\
\hline & -131.25 & & \\
\hline & -93.82 & -59.6325 & -1382.92 \\
\hline \multirow[t]{4}{*}{52} & 15.46 & & \\
\hline & -14.56 & & \\
\hline & -35.6 & & \\
\hline & -225.15 & -64.9625 & -1417.15 \\
\hline \multirow[t]{4}{*}{53} & -98.66 & & \\
\hline & 49.4 & & \\
\hline & -38.71 & & \\
\hline & -49.88 & -34.4625 & -1221.3 \\
\hline \multirow[t]{4}{*}{54} & -151.55 & & \\
\hline & 90.24 & & \\
\hline & -10.83 & & \\
\hline & 45.16 & -6.745 & -1043.31 \\
\hline \multirow[t]{4}{*}{55} & -63.88 & & \\
\hline & 22.86 & & \\
\hline & -71.02 & & \\
\hline & -117.37 & -57.3525 & -1368.28 \\
\hline \multirow[t]{4}{*}{56} & -152.92 & & \\
\hline & -54.28 & & \\
\hline & -26.24 & & \\
\hline & 12.79 & -55.1625 & -1354.22 \\
\hline \multirow[t]{4}{*}{57} & -193.31 & & \\
\hline & 140.53 & & \\
\hline & -166.06 & & \\
\hline & 72.98 & -36.465 & -1234.16 \\
\hline \multirow[t]{3}{*}{58} & 9.99 & & \\
\hline & -51.26 & & \\
\hline & -25.2 & & \\
\hline
\end{tabular}




\begin{tabular}{|c|c|c|c|}
\hline & -101.77 & -42.06 & -1270.09 \\
\hline \multirow[t]{4}{*}{59} & 68.6 & & \\
\hline & -108.29 & & \\
\hline & -28.77 & & \\
\hline & 14 & -13.615 & -1087.43 \\
\hline \multirow[t]{4}{*}{60} & 86.71 & & \\
\hline & -101.2 & & \\
\hline & -161.21 & & \\
\hline & -28.48 & -51.045 & -1327.78 \\
\hline SD & & 42.595746 & 273.525 \\
\hline AVERAGE & & -21.86538 & -1140.4 \\
\hline ppm D & \multicolumn{3}{|l|}{$\delta \mathrm{D}(\% \circ)$} \\
\hline 48.20463 & \multicolumn{3}{|l|}{638.7764} \\
\hline
\end{tabular}

Table S9. LOD-Limit of Detection (Data also used for LOQ)

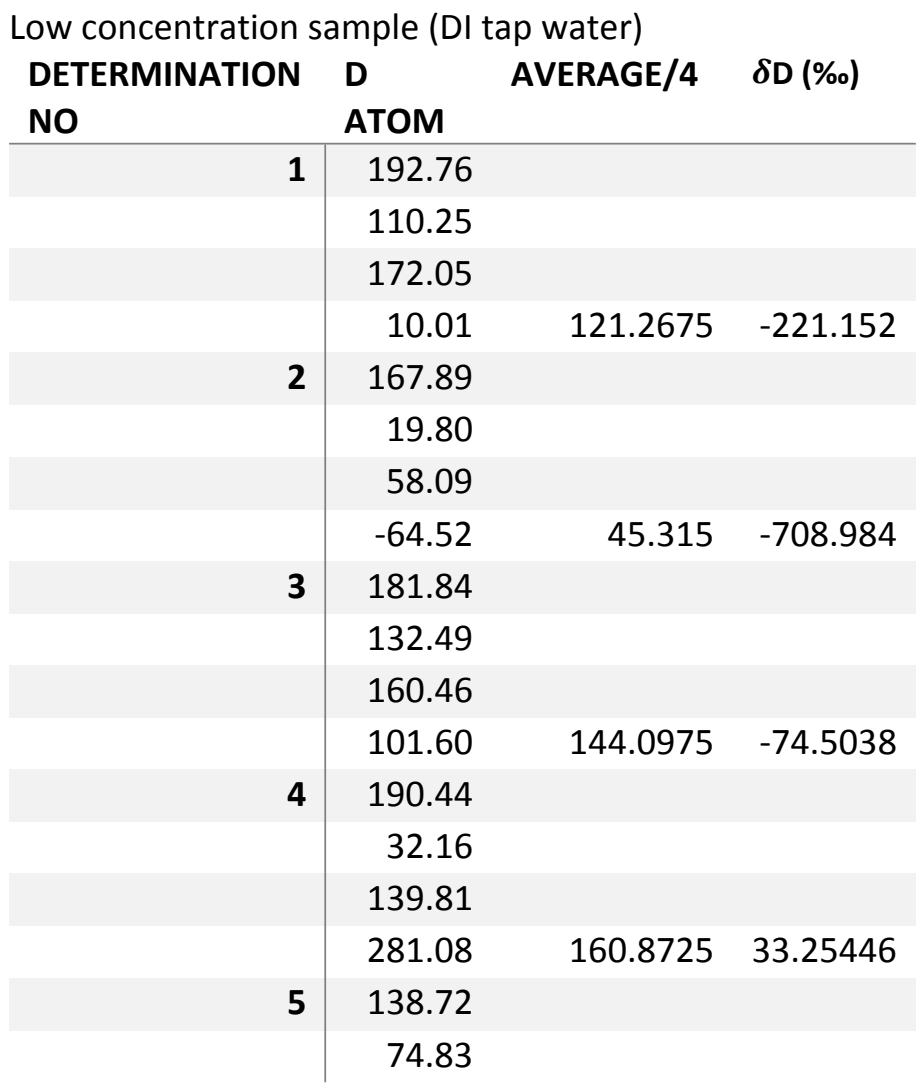




\begin{tabular}{|c|c|c|c|}
\hline & 43.14 & & \\
\hline & 54.10 & 77.6975 & -501.005 \\
\hline \multirow[t]{4}{*}{6} & 176.50 & & \\
\hline & 81.14 & & \\
\hline & 123.49 & & \\
\hline & 124.99 & 126.53 & -187.349 \\
\hline \multirow[t]{4}{*}{7} & -37.76 & & \\
\hline & -23.07 & & \\
\hline & 117.64 & & \\
\hline & 122.23 & 44.76 & -712.548 \\
\hline \multirow[t]{4}{*}{8} & -1.14 & & \\
\hline & 58.03 & & \\
\hline & 155.02 & & \\
\hline & 186.14 & 99.5125 & -360.889 \\
\hline \multirow[t]{4}{*}{9} & 118.70 & & \\
\hline & 149.97 & & \\
\hline & 80.90 & & \\
\hline & 233.27 & 145.71 & -64.1457 \\
\hline \multirow[t]{4}{*}{10} & 178.33 & & \\
\hline & 202.92 & & \\
\hline & 129.05 & & \\
\hline & 186.93 & 174.3075 & 119.56 \\
\hline \multirow[t]{4}{*}{11} & 70.17 & & \\
\hline & 195.72 & & \\
\hline & 32.32 & & \\
\hline & 154.08 & 113.0725 & -273.791 \\
\hline \multirow[t]{4}{*}{12} & 199.95 & & \\
\hline & 214.41 & & \\
\hline & 186.21 & & \\
\hline & 112.01 & 178.145 & 144.2123 \\
\hline \multirow[t]{4}{*}{13} & -28.53 & & \\
\hline & 127.10 & & \\
\hline & 52.94 & & \\
\hline & 214.24 & 91.4375 & -412.755 \\
\hline \multirow[t]{4}{*}{14} & 115.61 & & \\
\hline & 75.23 & & \\
\hline & -30.00 & & \\
\hline & 185.31 & 86.5375 & -444.227 \\
\hline \multirow[t]{4}{*}{15} & 169.65 & & \\
\hline & 141.98 & & \\
\hline & 244.41 & & \\
\hline & -3.94 & 138.025 & -113.511 \\
\hline \multirow[t]{4}{*}{16} & 270.61 & & \\
\hline & 32.41 & & \\
\hline & 284.60 & & \\
\hline & 172.11 & 189.9325 & 219.9369 \\
\hline
\end{tabular}




\begin{tabular}{|c|c|c|c|}
\hline \multirow[t]{4}{*}{17} & 115.26 & & \\
\hline & 54.17 & & \\
\hline & 240.14 & & \\
\hline & 206.97 & 154.135 & -10.0259 \\
\hline \multirow[t]{4}{*}{18} & 59.39 & & \\
\hline & 59.74 & & \\
\hline & 148.58 & & \\
\hline & 107.16 & 93.7175 & -398.11 \\
\hline \multirow[t]{4}{*}{19} & 91.56 & & \\
\hline & 297.16 & & \\
\hline & 178.47 & & \\
\hline & 205.96 & 193.2875 & 241.4903 \\
\hline \multirow[t]{4}{*}{20} & 119.75 & & \\
\hline & 201.81 & & \\
\hline & 145.20 & & \\
\hline & 199.05 & 166.4525 & 69.09974 \\
\hline \multirow[t]{4}{*}{21} & 211.98 & & \\
\hline & 140.32 & & \\
\hline & 169.10 & & \\
\hline & 115.46 & 159.215 & 22.60694 \\
\hline \multirow[t]{4}{*}{22} & 163.74 & & \\
\hline & 56.13 & & \\
\hline & 51.01 & & \\
\hline & -62.75 & 52.0325 & -665.841 \\
\hline \multirow[t]{4}{*}{23} & 145.00 & & \\
\hline & 174.29 & & \\
\hline & 224.35 & & \\
\hline & 165.91 & 177.3875 & 139.3461 \\
\hline \multirow[t]{4}{*}{24} & 280.64 & & \\
\hline & 125.62 & & \\
\hline & 223.87 & & \\
\hline & 205.06 & 208.7975 & 341.1322 \\
\hline \multirow[t]{4}{*}{25} & 43.99 & & \\
\hline & 309.86 & & \\
\hline & 206.39 & & \\
\hline & 117.66 & 169.475 & 88.5161 \\
\hline \multirow[t]{4}{*}{26} & 223.59 & & \\
\hline & 97.27 & & \\
\hline & 208.89 & & \\
\hline & 110.44 & 160.0475 & 27.95479 \\
\hline \multirow[t]{4}{*}{27} & 220.96 & & \\
\hline & 251.19 & & \\
\hline & 173.75 & & \\
\hline & 212.28 & 214.545 & 378.057 \\
\hline \multirow[t]{2}{*}{28} & 169.87 & & \\
\hline & 169.15 & & \\
\hline
\end{tabular}




\begin{tabular}{|c|c|c|c|}
\hline & 89.00 & & \\
\hline & 248.32 & 169.085 & 86.01075 \\
\hline \multirow[t]{4}{*}{29} & 166.08 & & \\
\hline & 85.05 & & \\
\hline & 196.06 & & \\
\hline & 189.84 & 159.2575 & 22.87996 \\
\hline \multirow[t]{4}{*}{30} & 298.06 & & \\
\hline & 224.01 & & \\
\hline & 139.22 & & \\
\hline & 26.93 & 172.055 & 105.09 \\
\hline \multirow[t]{4}{*}{31} & -66.62 & & \\
\hline & 187.86 & & \\
\hline & 239.51 & & \\
\hline & 208.35 & 142.275 & -86.2109 \\
\hline \multirow[t]{4}{*}{32} & 164.99 & & \\
\hline & 213.35 & & \\
\hline & 228.96 & & \\
\hline & 9.32 & 154.155 & -9.89746 \\
\hline \multirow[t]{4}{*}{33} & 157.94 & & \\
\hline & 160.58 & & \\
\hline & 268.18 & & \\
\hline & 148.40 & 183.775 & 180.38 \\
\hline \multirow[t]{4}{*}{34} & 199.80 & & \\
\hline & 17.87 & & \\
\hline & 177.57 & & \\
\hline & 22.03 & 104.3175 & -330.026 \\
\hline \multirow[t]{4}{*}{35} & 63.22 & & \\
\hline & 59.54 & & \\
\hline & 233.28 & & \\
\hline & 146.87 & 125.7275 & -192.504 \\
\hline \multirow[t]{4}{*}{36} & 236.74 & & \\
\hline & 96.58 & & \\
\hline & 152.26 & & \\
\hline & 315.78 & 200.34 & 286.7977 \\
\hline \multirow[t]{4}{*}{37} & 103.94 & & \\
\hline & 280.11 & & \\
\hline & 94.63 & & \\
\hline & 245.97 & 181.1625 & 163.597 \\
\hline \multirow[t]{4}{*}{38} & 216.79 & & \\
\hline & 53.49 & & \\
\hline & 138.72 & & \\
\hline & 217.66 & 156.665 & 6.226225 \\
\hline \multirow[t]{4}{*}{39} & 191.63 & & \\
\hline & 151.40 & & \\
\hline & 344.50 & & \\
\hline & 165.00 & 213.1325 & 368.9824 \\
\hline
\end{tabular}




\begin{tabular}{|c|c|c|c|}
\hline \multirow[t]{4}{*}{40} & 102.52 & & \\
\hline & 203.08 & & \\
\hline & 240.13 & & \\
\hline & 21.01 & 141.685 & -90.0008 \\
\hline \multirow[t]{4}{*}{41} & 230.30 & & \\
\hline & 248.69 & & \\
\hline & 132.78 & & \\
\hline & 241.32 & 213.2725 & 369.8818 \\
\hline \multirow[t]{4}{*}{42} & 137.81 & & \\
\hline & 169.27 & & \\
\hline & 172.73 & & \\
\hline & 168.78 & 162.1475 & 41.44488 \\
\hline \multirow[t]{4}{*}{43} & 134.42 & & \\
\hline & 255.55 & & \\
\hline & 94.23 & & \\
\hline & 146.73 & 157.7325 & 13.08363 \\
\hline \multirow[t]{4}{*}{44} & 116.15 & & \\
\hline & 166.67 & & \\
\hline & 160.71 & & \\
\hline & 215.65 & 164.795 & 58.45211 \\
\hline \multirow[t]{4}{*}{45} & 107.32 & & \\
\hline & 209.70 & & \\
\hline & 208.53 & & \\
\hline & 170.59 & 174.035 & 117.8095 \\
\hline \multirow[t]{4}{*}{46} & 509.98 & & \\
\hline & 130.10 & & \\
\hline & 85.77 & & \\
\hline & 111.55 & 209.35 & 344.6817 \\
\hline \multirow[t]{4}{*}{47} & 394.01 & & \\
\hline & 66.09 & & \\
\hline & 186.53 & & \\
\hline & 302.29 & 237.23 & 523.801 \\
\hline \multirow[t]{4}{*}{48} & 181.77 & & \\
\hline & 147.63 & & \\
\hline & 156.09 & & \\
\hline & 143.21 & 157.175 & 9.502362 \\
\hline \multirow[t]{4}{*}{49} & 276.30 & & \\
\hline & 217.84 & & \\
\hline & 394.40 & & \\
\hline & 136.97 & 256.3775 & 646.8228 \\
\hline \multirow[t]{4}{*}{50} & 136.55 & & \\
\hline & 443.70 & & \\
\hline & 177.40 & & \\
\hline & 172.08 & 232.4325 & 492.978 \\
\hline \multirow[t]{2}{*}{51} & 197.92 & & \\
\hline & 168.27 & & \\
\hline
\end{tabular}




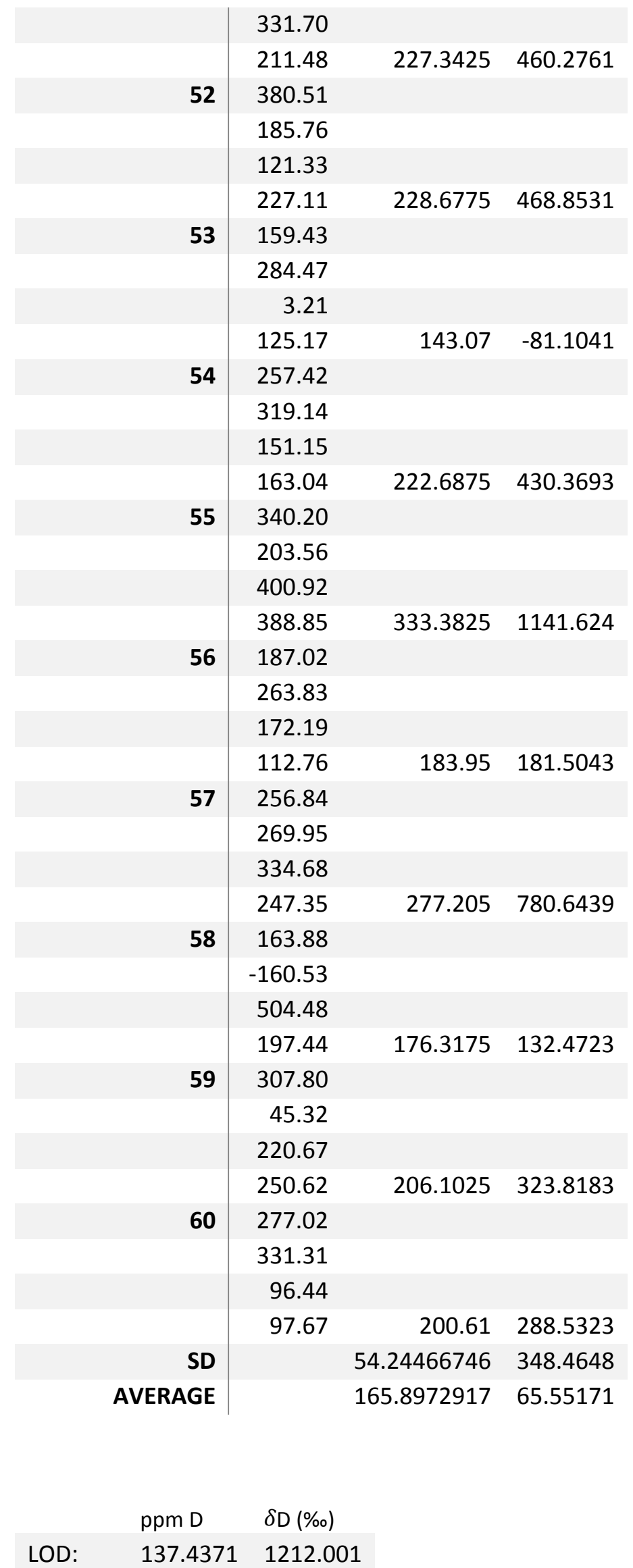


Table S10. Robustness- Temperature (Calibrated at $15.7^{\circ} \mathrm{C}$ )

\begin{tabular}{|c|c|c|c|c|}
\hline TEMP & NO & D ATOM & AVERAGE/4 & $\begin{array}{l}\% \\
\text { CHANGE } \\
\text { VS } \\
\text { TARGET }\end{array}$ \\
\hline \multirow[t]{12}{*}{13.7} & 1 & $4,905.95$ & & \\
\hline & & $4,933.08$ & & \\
\hline & & $5,058.20$ & & \\
\hline & & $4,950.07$ & 4961.825 & 0.47 \\
\hline & 2 & $4,936.31$ & & \\
\hline & & $5,001.91$ & & \\
\hline & & $4,761.30$ & & \\
\hline & & $4,949.86$ & 4912.345 & -0.53 \\
\hline & 3 & $4,884.50$ & & \\
\hline & & $4,918.27$ & & \\
\hline & & $4,817.24$ & & \\
\hline & & $4,807.86$ & 4856.9675 & -1.65 \\
\hline \multirow[t]{12}{*}{17.7} & 1 & $4,841.81$ & & \\
\hline & & $4,897.93$ & & \\
\hline & & $4,851.87$ & & \\
\hline & & $4,671.05$ & 4815.665 & -2.49 \\
\hline & 2 & $4,837.32$ & & \\
\hline & & $4,799.65$ & & \\
\hline & & $4,882.48$ & & \\
\hline & & $4,827.48$ & 4836.7325 & -2.06 \\
\hline & 3 & $4,849.02$ & & \\
\hline & & $4,591.67$ & & \\
\hline & & $4,914.72$ & & \\
\hline & & $4,840.82$ & 4799.0575 & -2.83 \\
\hline \multirow[t]{12}{*}{20.7} & 1 & $4,713.27$ & & \\
\hline & & $4,617.58$ & & \\
\hline & & $4,715.98$ & & \\
\hline & & $4,564.97$ & 4652.95 & -5.78 \\
\hline & 2 & $4,848.85$ & & \\
\hline & & $4,594.97$ & & \\
\hline & & $4,581.56$ & & \\
\hline & & $4,593.78$ & 4654.79 & -5.75 \\
\hline & 3 & $4,534.22$ & & \\
\hline & & $4,506.91$ & & \\
\hline & & $4,751.06$ & & \\
\hline & & $4,583.09$ & 4593.82 & -6.98 \\
\hline \multirow[t]{4}{*}{10.7} & 1 & $5,255.23$ & & \\
\hline & & $5,382.64$ & & \\
\hline & & $5,174.10$ & & \\
\hline & & $5,150.96$ & 5240.7325 & 6.12 \\
\hline
\end{tabular}


$2 \quad 5,255.31$

$5,333.68$

$5,288.50$

$\begin{array}{lll}5,305.87 & 5295.84 & 7.23\end{array}$

$3 \quad 5,213.91$

$5,132.07$

$5,177.21$

$5,213.05 \quad 5184.06 \quad 4.97$

Table S11. Robustness- $\mathrm{pH}$ (Calibrated at pH 7)

$\mathrm{pH}$ 2:

\begin{tabular}{|c|c|c|c|}
\hline DETERMINATION & READING & AVERAGE/4 & $\begin{array}{l}\% \text { CHANGE VS } \\
\text { TARGET }\end{array}$ \\
\hline \multirow[t]{4}{*}{1} & 5085.18 & & \\
\hline & 5043.65 & & \\
\hline & 4976.65 & & \\
\hline & 5112.71 & 5055 & 2.35 \\
\hline \multirow[t]{4}{*}{2} & 4947.91 & & \\
\hline & 4756.89 & & \\
\hline & 4992.60 & & \\
\hline & 4848.99 & 4887 & -1.05 \\
\hline \multirow[t]{4}{*}{3} & 5052.41 & & \\
\hline & 4960.29 & & \\
\hline & 4958.27 & & \\
\hline & 4930.92 & 4975 & 0.75 \\
\hline \multirow[t]{4}{*}{4} & 4890.77 & & \\
\hline & 4882.34 & & \\
\hline & 4925.41 & & \\
\hline & 4797.66 & 4874 & -1.31 \\
\hline
\end{tabular}




\begin{tabular}{l|l}
5 & 4878.58 \\
4739.66 \\
4905.20 \\
4977.34
\end{tabular}

$\begin{array}{lll}4977.34 & 4875 & -1.28\end{array}$

pH 10:

\begin{tabular}{|c|c|c|c|}
\hline DETERMINATION & READING & AVERAGE/4 & $\begin{array}{l}\% \text { CHANGE VS } \\
\text { TARGET }\end{array}$ \\
\hline \multirow[t]{4}{*}{1} & 4933.40 & & \\
\hline & 5268.89 & & \\
\hline & 4779.81 & & \\
\hline & 5050.82 & 5008 & 1.41 \\
\hline \multirow[t]{4}{*}{2} & 5067.45 & & \\
\hline & 5210.33 & & \\
\hline & 4954.10 & & \\
\hline & 4897.39 & 5032 & 1.90 \\
\hline \multirow[t]{4}{*}{3} & 4988.82 & & \\
\hline & 4691.61 & & \\
\hline & 5113.73 & & \\
\hline & 5195.96 & 4998 & 1.19 \\
\hline \multirow[t]{4}{*}{4} & 5167.37 & & \\
\hline & 5003.28 & & \\
\hline & 4850.69 & & \\
\hline & 5213.27 & 5059 & 2.43 \\
\hline \multirow[t]{4}{*}{5} & 4843.95 & & \\
\hline & 4902.21 & & \\
\hline & 5071.37 & & \\
\hline & 4888.75 & 4927 & -0.24 \\
\hline
\end{tabular}

Table S12. Robustness- Ionic Strength

$1 \% \mathrm{w} / \mathrm{v} \mathrm{NaCl}:$

\begin{tabular}{|c|c|c|c|}
\hline DETERMINATION & READING & AVERAGE/4 & $\begin{array}{l}\% \text { CHANGE VS } \\
\text { TARGET }\end{array}$ \\
\hline \multirow[t]{4}{*}{1} & $5,034.07$ & & \\
\hline & $4,860.83$ & & \\
\hline & $4,949.54$ & & \\
\hline & $5,008.00$ & 4963 & 0.50 \\
\hline \multirow[t]{4}{*}{2} & $4,937.57$ & & \\
\hline & $4,893.97$ & & \\
\hline & $4,866.99$ & & \\
\hline & $4,841.77$ & 4885 & -1.08 \\
\hline
\end{tabular}




\begin{tabular}{|c|c|c|c|}
\hline \multirow[t]{4}{*}{3} & \multicolumn{3}{|l|}{$4,855.72$} \\
\hline & \multicolumn{3}{|l|}{$4,960.03$} \\
\hline & \multicolumn{3}{|l|}{$4,963.68$} \\
\hline & $4,966.17$ & 4936 & -0.04 \\
\hline \multirow[t]{4}{*}{4} & $4,844.49$ & & \\
\hline & $5,058.37$ & & \\
\hline & $4,990.56$ & & \\
\hline & $5,046.61$ & 4985 & 0.94 \\
\hline \multirow[t]{4}{*}{5} & $4,994.84$ & & \\
\hline & $4,916.69$ & & \\
\hline & $5,046.41$ & & \\
\hline & $5,014.65$ & 4993 & 1.10 \\
\hline \multirow[t]{4}{*}{6} & $5,051.27$ & & \\
\hline & $4,936.58$ & & \\
\hline & $4,926.91$ & & \\
\hline & $4,987.80$ & 4976 & 0.75 \\
\hline \multirow[t]{4}{*}{7} & $20: 24: 00$ & & \\
\hline & $11: 45: 36$ & & \\
\hline & 07:12:00 & & \\
\hline & $15: 50: 24$ & 4973 & 0.69 \\
\hline \multirow[t]{4}{*}{8} & $19: 40: 48$ & & \\
\hline & 06:00:00 & & \\
\hline & $10: 48: 00$ & & \\
\hline & $08: 38: 24$ & 4996 & 1.17 \\
\hline \multirow[t]{4}{*}{9} & $19: 55: 12$ & & \\
\hline & $15: 21: 36$ & & \\
\hline & $11: 16: 48$ & & \\
\hline & $05: 45: 36$ & 5010 & 1.44 \\
\hline \multirow[t]{4}{*}{10} & $18: 43: 12$ & & \\
\hline & $21: 36: 00$ & & \\
\hline & $05: 16: 48$ & & \\
\hline & $22: 04: 48$ & 5032 & 1.88 \\
\hline \multirow[t]{4}{*}{11} & $06: 43: 12$ & & \\
\hline & 18:00:00 & & \\
\hline & $13: 26: 24$ & & \\
\hline & $18: 14: 24$ & 4932 & -0.14 \\
\hline \multirow[t]{4}{*}{12} & 10:19:12 & & \\
\hline & $5,016.25$ & & \\
\hline & $4,858.59$ & & \\
\hline & $4,976.79$ & 4984 & 0.91 \\
\hline \multirow[t]{4}{*}{13} & $5,038.36$ & & \\
\hline & $4,937.81$ & & \\
\hline & $4,886.92$ & & \\
\hline & $5,021.22$ & 4971 & 0.66 \\
\hline \multirow[t]{2}{*}{14} & $5,010.94$ & & \\
\hline & $5,119.51$ & & \\
\hline
\end{tabular}




\begin{tabular}{|c|c|c|c|}
\hline & 4,934.65 & & \\
\hline & $5,096.72$ & 5040 & 2.06 \\
\hline \multirow[t]{4}{*}{15} & $4,869.66$ & & \\
\hline & $5,104.08$ & & \\
\hline & 4,921.93 & & \\
\hline & 4,934.34 & 4958 & 0.38 \\
\hline \multirow[t]{4}{*}{16} & $5,008.22$ & & \\
\hline & $4,987.77$ & & \\
\hline & $4,981.38$ & & \\
\hline & $4,880.51$ & 4964 & 0.52 \\
\hline \multirow[t]{4}{*}{17} & $4,880.10$ & & \\
\hline & $5,062.46$ & & \\
\hline & $5,035.38$ & & \\
\hline & $4,958.78$ & 4984 & 0.92 \\
\hline \multirow[t]{4}{*}{18} & 4,936.19 & & \\
\hline & $4,983.59$ & & \\
\hline & $5,030.11$ & & \\
\hline & $4,941.56$ & 4973 & 0.69 \\
\hline \multirow[t]{4}{*}{19} & $5,036.56$ & & \\
\hline & $4,857.30$ & & \\
\hline & $4,891.72$ & & \\
\hline & $4,814.17$ & 4900 & -0.78 \\
\hline \multirow[t]{4}{*}{20} & $4,808.33$ & & \\
\hline & $4,954.01$ & & \\
\hline & $5,080.47$ & & \\
\hline & $5,127.86$ & 4993 & 1.09 \\
\hline
\end{tabular}

$6 \% \mathrm{w} / \mathrm{v} \mathrm{NaCl}:$

DETERMINATION READING AVERAGE/4 \% ChANGE VS

TARGET

\begin{tabular}{|c|c|c|c|}
\hline \multirow{5}{*}{$\begin{array}{l}1 \\
\end{array}$} & \multirow[b]{2}{*}{$5,302.11$} & \\
\hline & & & \\
\hline & $5,207.93$ & & \\
\hline & $4,942.07$ & & \\
\hline & $5,186.60$ & 5160 & 4.48 \\
\hline \multirow[t]{4}{*}{2} & $4,979.68$ & & \\
\hline & $4,902.16$ & & \\
\hline & $5,074.76$ & & \\
\hline & 4,987.76 & 4986 & 0.96 \\
\hline \multirow[t]{4}{*}{3} & $5,033.44$ & & \\
\hline & $5,002.23$ & & \\
\hline & $4,905.50$ & & \\
\hline & $5,046.25$ & 4997 & 1.18 \\
\hline \multirow[t]{2}{*}{4} & $4,901.42$ & & \\
\hline & $4,915.16$ & & \\
\hline
\end{tabular}




\begin{tabular}{|c|c|c|c|}
\hline & $4,607.94$ & & \\
\hline & $4,779.86$ & 4801 & -2.78 \\
\hline \multirow[t]{4}{*}{5} & $4,880.13$ & & \\
\hline & $4,915.04$ & & \\
\hline & $4,870.04$ & & \\
\hline & $4,822.98$ & 4872 & -1.35 \\
\hline \multirow[t]{4}{*}{6} & $4,962.92$ & & \\
\hline & $4,781.29$ & & \\
\hline & 4,797.09 & & \\
\hline & $4,886.56$ & 4857 & -1.65 \\
\hline \multirow[t]{4}{*}{7} & $4,771.28$ & & \\
\hline & $4,752.95$ & & \\
\hline & $5,011.36$ & & \\
\hline & $4,821.95$ & 4839 & -2.01 \\
\hline \multirow[t]{4}{*}{8} & $4,799.10$ & & \\
\hline & $4,811.52$ & & \\
\hline & $4,742.19$ & & \\
\hline & $4,884.74$ & 4809 & -2.62 \\
\hline \multirow[t]{4}{*}{9} & $4,855.57$ & & \\
\hline & $4,614.47$ & & \\
\hline & 4,959.19 & & \\
\hline & $4,607.83$ & 4759 & -3.63 \\
\hline \multirow[t]{4}{*}{10} & $4,723.82$ & & \\
\hline & $4,793.31$ & & \\
\hline & $4,681.06$ & & \\
\hline & $4,779.87$ & 4745 & -3.93 \\
\hline \multirow[t]{4}{*}{11} & $4,667.30$ & & \\
\hline & $4,819.23$ & & \\
\hline & $4,675.74$ & & \\
\hline & $4,802.64$ & 4741 & -4.00 \\
\hline \multirow[t]{4}{*}{12} & $4,761.42$ & & \\
\hline & $4,582.97$ & & \\
\hline & $4,632.02$ & & \\
\hline & $4,701.43$ & 4669 & -5.45 \\
\hline \multirow[t]{4}{*}{13} & $4,676.94$ & & \\
\hline & $4,648.59$ & & \\
\hline & $4,585.86$ & & \\
\hline & $4,682.28$ & 4648 & -5.88 \\
\hline \multirow[t]{4}{*}{14} & 4,519.98 & & \\
\hline & $4,542.92$ & & \\
\hline & $4,631.69$ & & \\
\hline & $4,728.32$ & 4606 & -6.74 \\
\hline \multirow[t]{4}{*}{15} & $4,609.95$ & & \\
\hline & $4,733.76$ & & \\
\hline & $4,576.02$ & & \\
\hline & 4,579.05 & 4625 & -6.36 \\
\hline
\end{tabular}




\begin{tabular}{|c|c|c|c|}
\hline \multirow{4}{*}{16} & \multicolumn{3}{|l|}{$4,763.09$} \\
\hline & \multicolumn{3}{|l|}{ 4,597.15 } \\
\hline & \multicolumn{3}{|l|}{$4,506.22$} \\
\hline & $4,582.72$ & 4612 & -6.61 \\
\hline \multirow[t]{4}{*}{17} & $4,563.28$ & & \\
\hline & $4,495.16$ & & \\
\hline & $4,601.02$ & & \\
\hline & $4,431.49$ & 4523 & -8.42 \\
\hline \multirow[t]{4}{*}{18} & 4,463.16 & & \\
\hline & $4,396.22$ & & \\
\hline & $4,521.54$ & & \\
\hline & 4,604.99 & 4496 & -8.95 \\
\hline \multirow[t]{4}{*}{19} & $4,501.29$ & & \\
\hline & $4,464.16$ & & \\
\hline & $4,574.02$ & & \\
\hline & $4,371.54$ & 4478 & -9.33 \\
\hline \multirow[t]{4}{*}{20} & $4,534.29$ & & \\
\hline & $4,558.77$ & & \\
\hline & $4,373.50$ & & \\
\hline & $4,714.64$ & 4545 & -7.96 \\
\hline
\end{tabular}

Table S13. Robustness- $\mathrm{N}_{2}$ Atmosphere

Remove flow of $\mathrm{N}_{2}$ :

\begin{tabular}{|c|c|c|c|}
\hline DETERMINATION & READING & AVERAGE/4 & $\begin{array}{l}\% \text { CHANGE VS } \\
\text { TARGET }\end{array}$ \\
\hline \multirow[t]{4}{*}{1} & $5,069.28$ & & \\
\hline & $4,792.33$ & & \\
\hline & $4,968.80$ & & \\
\hline & $4,954.58$ & 4946 & 0.15 \\
\hline \multirow[t]{4}{*}{2} & $4,896.89$ & & \\
\hline & $4,826.08$ & & \\
\hline & $5,005.54$ & & \\
\hline & $5,019.31$ & 4937 & -0.03 \\
\hline \multirow[t]{4}{*}{3} & $4,950.84$ & & \\
\hline & $4,890.86$ & & \\
\hline & $4,894.28$ & & \\
\hline & $5,043.17$ & 4945 & 0.12 \\
\hline \multirow[t]{4}{*}{4} & $5,056.37$ & & \\
\hline & $4,971.13$ & & \\
\hline & 4,847.39 & & \\
\hline & $5,101.52$ & 4994 & 1.12 \\
\hline
\end{tabular}




5 \begin{tabular}{r|r}
$4,978.98$ \\
$4,984.68$ \\
$5,022.97$ \\
$4,947.47$
\end{tabular}

4984

0.91

Expose to ambient atmosphere:

\begin{tabular}{|c|c|c|c|}
\hline DETERMINATION & READING & AVERAGE/4 & $\begin{array}{l}\% \text { CHANGE VS } \\
\text { TARGET }\end{array}$ \\
\hline \multirow[t]{4}{*}{1} & $5,074.70$ & & \\
\hline & $5,079.99$ & & \\
\hline & $4,944.45$ & & \\
\hline & $5,000.93$ & 5025 & 1.75 \\
\hline \multirow[t]{4}{*}{2} & $4,850.63$ & & \\
\hline & $4,877.02$ & & \\
\hline & $4,640.87$ & & \\
\hline & $5,064.48$ & 4858 & -1.63 \\
\hline \multirow[t]{4}{*}{3} & $5,033.49$ & & \\
\hline & $5,050.71$ & & \\
\hline & $5,046.37$ & & \\
\hline & $4,934.33$ & 5016 & 1.57 \\
\hline \multirow[t]{4}{*}{4} & $4,712.09$ & & \\
\hline & $4,837.68$ & & \\
\hline & $4,969.21$ & & \\
\hline & $5,010.34$ & 4882 & -1.14 \\
\hline \multirow[t]{4}{*}{5} & $4,742.75$ & & \\
\hline & $4,967.47$ & & \\
\hline & $4,970.06$ & & \\
\hline & $4,766.87$ & 4862 & -1.56 \\
\hline \multirow[t]{4}{*}{6} & $4,829.34$ & & \\
\hline & $4,765.93$ & & \\
\hline & $4,932.13$ & & \\
\hline & $4,818.90$ & 4837 & -2.07 \\
\hline \multirow[t]{4}{*}{7} & $4,921.36$ & & \\
\hline & $4,868.14$ & & \\
\hline & $4,812.30$ & & \\
\hline & $4,983.05$ & 4896 & -0.86 \\
\hline \multirow[t]{4}{*}{8} & $4,863.83$ & & \\
\hline & $4,912.29$ & & \\
\hline & $5,135.86$ & & \\
\hline & $4,818.07$ & 4933 & -0.12 \\
\hline \multirow[t]{4}{*}{9} & $4,803.51$ & & \\
\hline & $4,791.85$ & & \\
\hline & $5,058.01$ & & \\
\hline & $5,051.61$ & 4926 & -0.25 \\
\hline
\end{tabular}


10

4,916.26

4,877.19

$4,958.39$

4,823.10

11

$4,940.33$

4,966.71

$5,045.90$

4,858.99

$4,830.97$

$4,877.44$

$5,067.35$

$4,836.92$
4894

$-0.91$

4953

0.29

4903

$-0.72$

\section{Other Data}

Table S14. Simulation of Flow- $5 \mu \mathrm{l} / \mathrm{min}$ of 124504 ppm D standard

\begin{tabular}{r|rrrrr} 
MINUTE & \multicolumn{1}{l}{$\begin{array}{l}\text { H2O INITAL } \\
\text { CONC (PPM D) }\end{array}$} & $\begin{array}{l}\text { STD } \\
\text { ADDITION }\end{array}$ & $\begin{array}{l}\text { TOTAL } \\
\text { VOL }\end{array}$ & $\begin{array}{l}\text { NOMINAL } \\
\text { CONC. (PPM D) }\end{array}$ & \multicolumn{1}{l}{$\begin{array}{l}\text { READING } \\
\text { (PPM D) }\end{array}$} \\
\hline $\mathbf{1}$ & 156 & 0 & 5000 & 156 & 157.70 \\
$\mathbf{2}$ & 156 & 5 & 5005 & 280 & 345.39 \\
$\mathbf{3}$ & 156 & 10 & 5010 & 404 & 214.60 \\
$\mathbf{4}$ & 156 & 15 & 5015 & 528 & 260.08 \\
$\mathbf{5}$ & 156 & 20 & 5020 & 651 & 307.30 \\
$\mathbf{6}$ & 156 & 25 & 5025 & 775 & 551.87 \\
$\mathbf{7}$ & 156 & 30 & 5030 & 898 & 594.25 \\
$\mathbf{8}$ & 156 & 35 & 5035 & 1020 & 733.20 \\
$\mathbf{9}$ & 156 & 40 & 5040 & 1143 & 820.73 \\
$\mathbf{1 0}$ & 156 & 45 & 5045 & 1265 & 993.46 \\
$\mathbf{1 1}$ & 156 & 50 & 5050 & 1387 & $1,136.68$ \\
$\mathbf{1 2}$ & 156 & 55 & 5055 & 1509 & $1,111.57$ \\
$\mathbf{1 3}$ & 156 & 60 & 5060 & 1630 & $1,643.59$ \\
$\mathbf{1 4}$ & 156 & 65 & 5065 & 1752 & $1,319.69$ \\
$\mathbf{1 5}$ & 156 & 70 & 5070 & 1873 & $1,477.45$
\end{tabular}




\begin{tabular}{|c|c|c|c|c|c|}
\hline 16 & 156 & 75 & 5075 & 1994 & $1,976.97$ \\
\hline 17 & 156 & 80 & 5080 & 2114 & $1,876.35$ \\
\hline 18 & 156 & 85 & 5085 & 2235 & $2,066.05$ \\
\hline 19 & 156 & 90 & 5090 & 2355 & $2,209.34$ \\
\hline 20 & 156 & 95 & 5095 & 2475 & 2,229.99 \\
\hline 21 & 156 & 100 & 5100 & 2594 & $2,327.11$ \\
\hline 22 & 156 & 105 & 5105 & 2714 & $2,326.65$ \\
\hline 23 & 156 & 110 & 5110 & 2833 & $2,543.14$ \\
\hline 24 & 156 & 115 & 5115 & 2952 & $2,677.20$ \\
\hline 25 & 156 & 120 & 5120 & 3070 & $2,720.19$ \\
\hline 26 & 156 & 125 & 5125 & 3189 & $2,693.47$ \\
\hline 27 & 156 & 130 & 5130 & 3307 & $3,134.83$ \\
\hline 28 & 156 & 135 & 5135 & 3425 & $3,114.22$ \\
\hline 29 & 156 & 140 & 5140 & 3543 & $3,109.41$ \\
\hline 30 & 156 & 145 & 5145 & 3660 & $3,676.68$ \\
\hline 31 & 156 & 150 & 5150 & 3778 & $3,658.79$ \\
\hline 32 & 156 & 155 & 5155 & 3895 & $3,633.67$ \\
\hline 33 & 156 & 160 & 5160 & 4012 & $3,732.01$ \\
\hline 34 & 156 & 165 & 5165 & 4128 & $4,191.92$ \\
\hline 35 & 156 & 170 & 5170 & 4245 & $3,972.07$ \\
\hline 36 & 156 & 175 & 5175 & 4361 & $4,075.31$ \\
\hline 37 & 156 & 180 & 5180 & 4477 & $4,457.20$ \\
\hline 38 & 156 & 185 & 5185 & 4593 & $4,410.49$ \\
\hline 39 & 156 & 190 & 5190 & 4708 & $4,551.67$ \\
\hline 40 & 156 & 195 & 5195 & 4824 & $4,706.09$ \\
\hline 41 & 156 & 200 & 5200 & 4939 & $4,950.64$ \\
\hline 42 & 156 & 205 & 5205 & 5053 & $4,979.69$ \\
\hline 43 & 156 & 210 & 5210 & 5168 & $5,171.17$ \\
\hline 44 & 156 & 215 & 5215 & 5283 & $5,208.28$ \\
\hline 45 & 156 & 220 & 5220 & 5397 & $5,339.49$ \\
\hline 46 & 156 & 225 & 5225 & 5511 & $5,429.01$ \\
\hline 47 & 156 & 230 & 5230 & 5624 & $5,320.15$ \\
\hline 48 & 156 & 235 & 5235 & 5738 & $5,806.03$ \\
\hline 49 & 156 & 240 & 5240 & 5851 & $5,844.01$ \\
\hline 50 & 156 & 245 & 5245 & 5964 & $5,881.31$ \\
\hline 51 & 156 & 250 & 5250 & 6077 & $5,911.47$ \\
\hline 52 & 156 & 255 & 5255 & 6190 & $6,296.50$ \\
\hline 53 & 156 & 260 & 5260 & 6302 & $6,295.81$ \\
\hline 54 & 156 & 265 & 5265 & 6415 & $6,226.95$ \\
\hline 55 & 156 & 270 & 5270 & 6527 & $6,342.84$ \\
\hline 56 & 156 & 275 & 5275 & 6639 & $6,592.36$ \\
\hline 57 & 156 & 280 & 5280 & 6750 & $6,834.26$ \\
\hline 58 & 156 & 285 & 5285 & 6862 & $6,603.12$ \\
\hline 59 & 156 & 290 & 5290 & 6973 & $6,790.72$ \\
\hline 60 & 156 & 295 & 5295 & 7084 & $6,754.42$ \\
\hline 61 & 156 & 300 & 5300 & 7195 & $7,159.72$ \\
\hline
\end{tabular}




\begin{tabular}{|c|c|c|c|c|c|}
\hline 62 & 156 & 305 & 5305 & 7305 & $7,189.00$ \\
\hline 63 & 156 & 310 & 5310 & 7415 & $7,177.49$ \\
\hline 64 & 156 & 315 & 5315 & 7526 & $7,554.58$ \\
\hline 65 & 156 & 320 & 5320 & 7636 & $7,662.91$ \\
\hline 66 & 156 & 325 & 5325 & 7745 & $7,717.08$ \\
\hline 67 & 156 & 330 & 5330 & 7855 & $7,717.46$ \\
\hline 68 & 156 & 335 & 5335 & 7964 & $8,013.22$ \\
\hline 69 & 156 & 340 & 5340 & 8073 & $8,140.51$ \\
\hline 70 & 156 & 345 & 5345 & 8182 & $8,040.18$ \\
\hline 71 & 156 & 350 & 5350 & 8291 & $8,042.80$ \\
\hline 72 & 156 & 355 & 5355 & 8399 & $8,328.32$ \\
\hline 73 & 156 & 360 & 5360 & 8508 & $8,436.88$ \\
\hline 74 & 156 & 365 & 5365 & 8616 & $8,395.91$ \\
\hline 75 & 156 & 370 & 5370 & 8724 & $8,715.83$ \\
\hline 76 & 156 & 375 & 5375 & 8831 & $8,810.69$ \\
\hline 77 & 156 & 380 & 5380 & 8939 & $8,941.18$ \\
\hline 78 & 156 & 385 & 5385 & 9046 & $8,991.98$ \\
\hline 79 & 156 & 390 & 5390 & 9153 & $9,080.46$ \\
\hline 80 & 156 & 395 & 5395 & 9260 & $9,225.52$ \\
\hline 81 & 156 & 400 & 5400 & 9367 & $9,405.35$ \\
\hline 82 & 156 & 405 & 5405 & 9473 & $9,348.27$ \\
\hline 83 & 156 & 410 & 5410 & 9580 & $9,442.59$ \\
\hline 84 & 156 & 415 & 5415 & 9686 & $9,550.20$ \\
\hline 85 & 156 & 420 & 5420 & 9792 & $9,769.19$ \\
\hline 86 & 156 & 425 & 5425 & 9898 & $9,859.54$ \\
\hline 87 & 156 & 430 & 5430 & 10003 & $9,900.22$ \\
\hline 88 & 156 & 435 & 5435 & 10108 & $9,966.07$ \\
\hline 89 & 156 & 440 & 5440 & 10214 & $10,186.93$ \\
\hline 90 & 156 & 445 & 5445 & 10319 & $10,135.74$ \\
\hline 91 & 156 & 450 & 5450 & 10423 & $10,270.01$ \\
\hline 92 & 156 & 455 & 5455 & 10528 & $10,629.71$ \\
\hline 93 & 156 & 460 & 5460 & 10632 & $10,475.57$ \\
\hline 94 & 156 & 465 & 5465 & 10736 & $10,675.54$ \\
\hline 95 & 156 & 470 & 5470 & 10840 & $10,902.52$ \\
\hline 96 & 156 & 475 & 5475 & 10944 & $11,015.99$ \\
\hline 97 & 156 & 480 & 5480 & 11048 & $11,014.75$ \\
\hline 98 & 156 & 485 & 5485 & 11151 & $11,479.00$ \\
\hline 99 & 156 & 490 & 5490 & 11254 & $11,282.92$ \\
\hline 100 & 156 & 495 & 5495 & 11358 & $11,394.03$ \\
\hline 101 & 156 & 500 & 5500 & 11460 & $11,482.33$ \\
\hline 102 & 156 & 505 & 5505 & 11563 & $11,667.82$ \\
\hline 103 & 156 & 510 & 5510 & 11666 & $11,946.58$ \\
\hline 104 & 156 & 515 & 5515 & 11768 & $11,825.07$ \\
\hline 105 & 156 & 520 & 5520 & 11870 & $11,684.90$ \\
\hline 106 & 156 & 525 & 5525 & 11972 & $12,034.82$ \\
\hline 107 & 156 & 530 & 5530 & 12074 & $12,239.77$ \\
\hline
\end{tabular}




\begin{tabular}{|c|c|c|c|c|c|}
\hline 108 & 156 & 535 & 5535 & 12175 & $12,044.41$ \\
\hline 109 & 156 & 540 & 5540 & 12277 & $12,328.49$ \\
\hline 110 & 156 & 545 & 5545 & 12378 & $12,200.00$ \\
\hline 111 & 156 & 550 & 5550 & 12479 & $12,693.46$ \\
\hline 112 & 156 & 555 & 5555 & 12580 & $12,583.69$ \\
\hline 113 & 156 & 560 & 5560 & 12680 & $12,578.40$ \\
\hline 114 & 156 & 565 & 5565 & 12781 & $12,566.16$ \\
\hline 115 & 156 & 570 & 5570 & 12881 & $12,766.10$ \\
\hline 116 & 156 & 575 & 5575 & 12981 & $12,986.02$ \\
\hline 117 & 156 & 580 & 5580 & 13081 & $12,902.04$ \\
\hline 118 & 156 & 585 & 5585 & 13181 & $13,038.81$ \\
\hline 119 & 156 & 590 & 5590 & 13280 & $13,199.59$ \\
\hline 120 & 156 & 595 & 5595 & 13380 & $13,362.72$ \\
\hline 121 & 156 & 600 & 5600 & 13479 & $13,463.44$ \\
\hline 122 & 156 & 605 & 5605 & 13578 & $13,436.32$ \\
\hline 123 & 156 & 610 & 5610 & 13677 & $13,731.79$ \\
\hline 124 & 156 & 615 & 5615 & 13776 & $13,874.96$ \\
\hline 125 & 156 & 620 & 5620 & 13874 & $13,900.52$ \\
\hline 126 & 156 & 625 & 5625 & 13972 & $13,902.06$ \\
\hline 127 & 156 & 630 & 5630 & 14071 & $13,885.46$ \\
\hline 128 & 156 & 635 & 5635 & 14169 & $14,259.21$ \\
\hline 129 & 156 & 640 & 5640 & 14266 & $14,234.74$ \\
\hline 130 & 156 & 645 & 5645 & 14364 & $14,222.81$ \\
\hline 131 & 156 & 650 & 5650 & 14462 & $14,446.50$ \\
\hline 132 & 156 & 655 & 5655 & 14559 & $14,674.86$ \\
\hline 133 & 156 & 660 & 5660 & 14656 & $14,619.14$ \\
\hline 134 & 156 & 665 & 5665 & 14753 & $14,585.80$ \\
\hline 135 & 156 & 670 & 5670 & 14850 & $14,707.07$ \\
\hline 136 & 156 & 675 & 5675 & 14946 & $15,012.03$ \\
\hline 137 & 156 & 680 & 5680 & 15043 & $15,064.97$ \\
\hline 138 & 156 & 685 & 5685 & 15139 & $15,021.21$ \\
\hline 139 & 156 & 690 & 5690 & 15235 & $15,016.95$ \\
\hline 140 & 156 & 695 & 5695 & 15331 & $15,432.44$ \\
\hline 141 & 156 & 700 & 5700 & 15427 & $15,386.34$ \\
\hline 142 & 156 & 705 & 5705 & 15522 & $15,374.50$ \\
\hline 143 & 156 & 710 & 5710 & 15618 & $15,418.42$ \\
\hline 144 & 156 & 715 & 5715 & 15713 & $15,919.40$ \\
\hline 145 & 156 & 720 & 5720 & 15808 & $15,790.17$ \\
\hline 146 & 156 & 725 & 5725 & 15903 & $15,755.14$ \\
\hline 147 & 156 & 730 & 5730 & 15998 & $15,907.44$ \\
\hline 148 & 156 & 735 & 5735 & 16092 & $16,130.88$ \\
\hline 149 & 156 & 740 & 5740 & 16187 & $16,070.65$ \\
\hline 150 & 156 & 745 & 5745 & 16281 & $16,175.83$ \\
\hline 151 & 156 & 750 & 5750 & 16375 & $16,176.09$ \\
\hline 152 & 156 & 755 & 5755 & 16469 & $16,529.58$ \\
\hline 153 & 156 & 760 & 5760 & 16563 & $16,456.97$ \\
\hline
\end{tabular}




\begin{tabular}{|c|c|c|c|c|c|}
\hline 154 & 156 & 765 & 5765 & 16657 & $16,617.42$ \\
\hline 155 & 156 & 770 & 5770 & 16750 & $16,695.59$ \\
\hline 156 & 156 & 775 & 5775 & 16843 & $16,916.00$ \\
\hline 157 & 156 & 780 & 5780 & 16937 & $16,868.47$ \\
\hline 158 & 156 & 785 & 5785 & 17030 & $16,830.69$ \\
\hline 159 & 156 & 790 & 5790 & 17122 & $17,049.49$ \\
\hline 160 & 156 & 795 & 5795 & 17215 & $17,364.42$ \\
\hline 161 & 156 & 800 & 5800 & 17307 & $17,392.23$ \\
\hline 162 & 156 & 805 & 5805 & 17400 & $17,373.31$ \\
\hline 163 & 156 & 810 & 5810 & 17492 & $17,527.28$ \\
\hline 164 & 156 & 815 & 5815 & 17584 & $17,738.02$ \\
\hline 165 & 156 & 820 & 5820 & 17676 & $17,719.89$ \\
\hline 166 & 156 & 825 & 5825 & 17768 & $17,567.05$ \\
\hline 167 & 156 & 830 & 5830 & 17859 & $17,655.86$ \\
\hline 168 & 156 & 835 & 5835 & 17950 & $17,991.59$ \\
\hline 169 & 156 & 840 & 5840 & 18042 & $18,114.18$ \\
\hline 170 & 156 & 845 & 5845 & 18133 & $18,114.71$ \\
\hline 171 & 156 & 850 & 5850 & 18224 & $18,128.97$ \\
\hline 172 & 156 & 855 & 5855 & 18314 & $18,410.17$ \\
\hline 173 & 156 & 860 & 5860 & 18405 & $18,508.58$ \\
\hline 174 & 156 & 865 & 5865 & 18495 & $18,389.44$ \\
\hline 175 & 156 & 870 & 5870 & 18586 & $18,455.94$ \\
\hline 176 & 156 & 875 & 5875 & 18676 & $18,799.74$ \\
\hline 177 & 156 & 880 & 5880 & 18766 & $18,831.26$ \\
\hline 178 & 156 & 885 & 5885 & 18856 & $18,800.37$ \\
\hline 179 & 156 & 890 & 5890 & 18945 & $18,695.47$ \\
\hline 180 & 156 & 895 & 5895 & 19035 & $19,198.74$ \\
\hline 181 & 156 & 900 & 5900 & 19124 & $19,224.67$ \\
\hline 182 & 156 & 905 & 5905 & 19214 & $18,984.06$ \\
\hline 183 & 156 & 910 & 5910 & 19303 & $19,146.88$ \\
\hline 184 & 156 & 915 & 5915 & 19392 & $19,354.22$ \\
\hline 185 & 156 & 920 & 5920 & 19480 & $19,396.15$ \\
\hline 186 & 156 & 925 & 5925 & 19569 & $19,520.23$ \\
\hline 187 & 156 & 930 & 5930 & 19657 & $19,557.82$ \\
\hline 188 & 156 & 935 & 5935 & 19746 & $19,857.47$ \\
\hline 189 & 156 & 940 & 5940 & 19834 & $19,803.22$ \\
\hline 190 & 156 & 945 & 5945 & 19922 & $19,794.26$ \\
\hline 191 & 156 & 950 & 5950 & 20010 & $20,183.90$ \\
\hline 192 & 156 & 955 & 5955 & 20098 & $20,182.77$ \\
\hline 193 & 156 & 960 & 5960 & 20185 & $20,225.89$ \\
\hline 194 & 156 & 965 & 5965 & 20273 & $20,196.95$ \\
\hline 195 & 156 & 970 & 5970 & 20360 & $20,374.35$ \\
\hline 196 & 156 & 975 & 5975 & 20447 & $20,414.20$ \\
\hline 197 & 156 & 980 & 5980 & 20534 & $20,472.88$ \\
\hline 198 & 156 & 985 & 5985 & 20621 & $20,748.91$ \\
\hline 199 & 156 & 990 & 5990 & 20708 & $20,818.49$ \\
\hline
\end{tabular}


\begin{tabular}{l|lllll}
200 & 156 & 995 & 5995 & 20794 & $20,969.09$
\end{tabular}

\section{Signal Amplification - EtOH with 156 -1143 ppm D Calibration}

The origin of the analytical noise is twofold; while the $\mathrm{H} 2 \mathrm{O}$ and HDO peaks are reasonably well resolved from each other, the resolution is not complete, and this reduces the quality of the calibration and therefore the quantification. The second cause is that the HDO scissoring peak used in this method is relatively small; a good comparison is to the O-D stretching absorbance shown in spectrum A of Figure $1(2480 \mathrm{~cm}-1)$. If a peak is small even at high concentration, as is the case for all the scissoring absorbances shown in spectrum C of Figure 1 , then the response of the peak to changes in concentration will also be small. The response of a peak to analyte concentration is directly proportional to the sensitivity of a method relying on it, so the scope for improving sensitivity for a small peak is limited.

Table S15. Signal amplification with EtOH. $156-1143$ D calibration.

\begin{tabular}{lr|rr}
$\begin{array}{lll}\text { DET } \\
\text { NO. }\end{array}$ & READING & AVERAGE/4 \\
\hline & $\mathbf{1}$ & $1,160.32$ & \\
& & $1,152.53$ & \\
& & $1,147.14$ & \\
& $\mathbf{2}$ & $1,157.18$ & 1154.293 \\
& & $1,144.09$ & \\
& & $1,160.03$ & \\
& & $1,150.77$ & \\
& $\mathbf{3}$ & $1,158.14$ & 1153.258 \\
& & $1,163.50$ & \\
& & $1,149.45$ & \\
& & $1,154.17$ & \\
& $\mathbf{4}$ & $1,156.43$ & 1156.888 \\
& $1,152.99$ & \\
& $1,167.16$ & \\
& & $1,162.56$ & 1159.785 \\
& $\mathbf{5}$ & $1,156.85$ & \\
& $1,168.45$ & \\
& &
\end{tabular}




\begin{tabular}{|c|c|c|}
\hline & $1,150.77$ & \\
\hline & $1,163.50$ & 1159.893 \\
\hline \multirow[t]{4}{*}{6} & $1,154.65$ & \\
\hline & $1,158.51$ & \\
\hline & $1,148.34$ & \\
\hline & $1,154.01$ & 1153.878 \\
\hline \multirow[t]{4}{*}{7} & $1,151.61$ & \\
\hline & $1,165.42$ & \\
\hline & $1,156.15$ & \\
\hline & $1,173.10$ & 1161.57 \\
\hline \multirow[t]{4}{*}{8} & $1,155.83$ & \\
\hline & $1,155.83$ & \\
\hline & $1,159.23$ & \\
\hline & $1,157.47$ & 1157.09 \\
\hline \multirow[t]{4}{*}{9} & $1,167.03$ & \\
\hline & $1,154.69$ & \\
\hline & $1,158.55$ & \\
\hline & $1,145.04$ & 1156.328 \\
\hline \multirow[t]{4}{*}{10} & $1,149.20$ & \\
\hline & $1,157.95$ & \\
\hline & $1,165.40$ & \\
\hline & $1,151.09$ & 1155.91 \\
\hline \multirow[t]{4}{*}{11} & $1,150.53$ & \\
\hline & $1,149.86$ & \\
\hline & $1,156.84$ & \\
\hline & $1,155.48$ & 1153.178 \\
\hline \multirow[t]{4}{*}{12} & $1,146.31$ & \\
\hline & $1,153.69$ & \\
\hline & $1,165.20$ & \\
\hline & $1,157.96$ & 1155.79 \\
\hline \multirow[t]{4}{*}{13} & $1,153.05$ & \\
\hline & $1,158.13$ & \\
\hline & $1,154.56$ & \\
\hline & $1,157.78$ & 1155.88 \\
\hline \multirow[t]{4}{*}{14} & $1,145.03$ & \\
\hline & $1,159.64$ & \\
\hline & $1,149.13$ & \\
\hline & $1,147.72$ & 1150.38 \\
\hline \multirow[t]{4}{*}{15} & $1,157.44$ & \\
\hline & $1,161.92$ & \\
\hline & $1,149.96$ & \\
\hline & $1,155.84$ & 1156.29 \\
\hline \multirow[t]{4}{*}{16} & $1,148.57$ & \\
\hline & $1,160.07$ & \\
\hline & $1,138.45$ & \\
\hline & $1,148.03$ & 1148.78 \\
\hline
\end{tabular}




\begin{tabular}{|c|c|c|}
\hline \multirow[t]{4}{*}{17} & \multicolumn{2}{|l|}{$1,150.82$} \\
\hline & \multicolumn{2}{|l|}{$1,155.88$} \\
\hline & \multicolumn{2}{|l|}{$1,153.87$} \\
\hline & $1,159.57$ & 1155.035 \\
\hline \multirow[t]{4}{*}{18} & $1,141.70$ & \\
\hline & $1,148.53$ & \\
\hline & $1,143.91$ & \\
\hline & $1,153.33$ & 1146.868 \\
\hline \multirow[t]{4}{*}{19} & $1,150.11$ & \\
\hline & $1,162.65$ & \\
\hline & $1,150.41$ & \\
\hline & $1,148.52$ & 1152.923 \\
\hline \multirow[t]{4}{*}{20} & $1,155.86$ & \\
\hline & $1,140.72$ & \\
\hline & $1,152.93$ & \\
\hline & $1,151.58$ & 1150.273 \\
\hline \multirow[t]{4}{*}{21} & $1,158.87$ & \\
\hline & $1,149.81$ & \\
\hline & $1,138.86$ & \\
\hline & $1,154.21$ & 1150.438 \\
\hline \multirow[t]{4}{*}{22} & $1,164.27$ & \\
\hline & $1,152.00$ & \\
\hline & $1,152.59$ & \\
\hline & $1,152.01$ & 1155.218 \\
\hline \multirow[t]{4}{*}{23} & $1,156.20$ & \\
\hline & $1,159.76$ & \\
\hline & $1,152.75$ & \\
\hline & $1,152.72$ & 1155.358 \\
\hline \multirow[t]{4}{*}{24} & $1,150.45$ & \\
\hline & $1,155.55$ & \\
\hline & $1,155.21$ & \\
\hline & $1,154.83$ & 1154.01 \\
\hline \multirow[t]{4}{*}{25} & $1,153.80$ & \\
\hline & $1,162.67$ & \\
\hline & $1,157.93$ & \\
\hline & $1,143.59$ & 1154.498 \\
\hline \multirow[t]{4}{*}{26} & $1,154.24$ & \\
\hline & $1,161.72$ & \\
\hline & $1,153.86$ & \\
\hline & $1,155.88$ & 1156.425 \\
\hline \multirow[t]{4}{*}{27} & $1,142.96$ & \\
\hline & $1,159.41$ & \\
\hline & $1,153.95$ & \\
\hline & $1,142.72$ & 1149.76 \\
\hline \multirow[t]{2}{*}{28} & $1,157.58$ & \\
\hline & $1,160.81$ & \\
\hline
\end{tabular}




\begin{tabular}{|c|c|c|}
\hline & $1,151.14$ & \\
\hline & $1,151.88$ & 1155.353 \\
\hline \multirow[t]{4}{*}{29} & $1,134.94$ & \\
\hline & $1,150.62$ & \\
\hline & $1,147.60$ & \\
\hline & $1,151.26$ & 1146.105 \\
\hline \multirow[t]{4}{*}{30} & $1,160.17$ & \\
\hline & $1,157.71$ & \\
\hline & $1,143.11$ & \\
\hline & $1,149.88$ & 1152.718 \\
\hline \multirow[t]{4}{*}{31} & $1,149.05$ & \\
\hline & $1,148.47$ & \\
\hline & $1,155.48$ & \\
\hline & $1,145.09$ & 1149.523 \\
\hline \multirow[t]{4}{*}{32} & $1,157.78$ & \\
\hline & $1,157.53$ & \\
\hline & $1,154.49$ & \\
\hline & $1,152.76$ & 1155.64 \\
\hline \multirow[t]{4}{*}{33} & $1,156.25$ & \\
\hline & $1,153.26$ & \\
\hline & $1,157.02$ & \\
\hline & $1,153.55$ & 1155.02 \\
\hline \multirow[t]{4}{*}{34} & $1,151.99$ & \\
\hline & $1,151.82$ & \\
\hline & $1,155.26$ & \\
\hline & $1,147.87$ & 1151.735 \\
\hline \multirow[t]{4}{*}{35} & $1,154.62$ & \\
\hline & $1,163.70$ & \\
\hline & $1,142.23$ & \\
\hline & $1,156.91$ & 1154.365 \\
\hline \multirow[t]{4}{*}{36} & $1,161.54$ & \\
\hline & $1,149.03$ & \\
\hline & $1,160.16$ & \\
\hline & $1,154.74$ & 1156.368 \\
\hline \multirow[t]{4}{*}{37} & $1,155.17$ & \\
\hline & $1,154.80$ & \\
\hline & $1,157.54$ & \\
\hline & $1,172.76$ & 1160.068 \\
\hline \multirow[t]{4}{*}{38} & $1,146.35$ & \\
\hline & $1,146.53$ & \\
\hline & $1,146.79$ & \\
\hline & $1,152.92$ & 1148.148 \\
\hline \multirow[t]{4}{*}{39} & $1,145.11$ & \\
\hline & $1,151.51$ & \\
\hline & $1,155.13$ & \\
\hline & $1,156.11$ & 1151.965 \\
\hline
\end{tabular}




\begin{tabular}{|c|c|c|}
\hline \multirow[t]{4}{*}{40} & \multicolumn{2}{|l|}{$1,159.62$} \\
\hline & \multicolumn{2}{|l|}{$1,149.68$} \\
\hline & \multicolumn{2}{|l|}{$1,158.47$} \\
\hline & $1,149.02$ & 1154.198 \\
\hline \multirow[t]{4}{*}{41} & $1,144.04$ & \\
\hline & $1,164.98$ & \\
\hline & $1,158.38$ & \\
\hline & $1,149.29$ & 1154.173 \\
\hline \multirow[t]{4}{*}{42} & $1,168.08$ & \\
\hline & $1,163.59$ & \\
\hline & $1,155.42$ & \\
\hline & $1,147.31$ & 1158.6 \\
\hline \multirow[t]{4}{*}{43} & $1,162.06$ & \\
\hline & $1,157.54$ & \\
\hline & $1,151.22$ & \\
\hline & $1,139.41$ & 1152.558 \\
\hline \multirow[t]{4}{*}{44} & $1,145.45$ & \\
\hline & $1,151.59$ & \\
\hline & $1,156.64$ & \\
\hline & $1,146.77$ & 1150.113 \\
\hline \multirow[t]{4}{*}{45} & $1,163.71$ & \\
\hline & $1,141.93$ & \\
\hline & $1,142.76$ & \\
\hline & $1,150.78$ & 1149.795 \\
\hline \multirow[t]{4}{*}{46} & $1,150.33$ & \\
\hline & $1,147.93$ & \\
\hline & $1,151.79$ & \\
\hline & $1,158.08$ & 1152.033 \\
\hline \multirow[t]{4}{*}{47} & $1,154.22$ & \\
\hline & $1,146.98$ & \\
\hline & $1,155.62$ & \\
\hline & $1,172.15$ & 1157.243 \\
\hline \multirow[t]{4}{*}{48} & $1,157.40$ & \\
\hline & $1,153.55$ & \\
\hline & $1,166.60$ & \\
\hline & $1,154.02$ & 1157.893 \\
\hline \multirow[t]{4}{*}{49} & $1,151.85$ & \\
\hline & $1,151.94$ & \\
\hline & $1,159.89$ & \\
\hline & $1,159.90$ & 1155.895 \\
\hline \multirow[t]{4}{*}{50} & $1,168.57$ & \\
\hline & $1,161.60$ & \\
\hline & $1,161.01$ & \\
\hline & $1,133.41$ & 1156.148 \\
\hline \multirow[t]{2}{*}{51} & $1,159.02$ & \\
\hline & $1,163.87$ & \\
\hline
\end{tabular}




\begin{tabular}{|c|c|c|}
\hline & $1,146.30$ & \\
\hline & $1,160.94$ & 1157.533 \\
\hline \multirow[t]{4}{*}{52} & $1,153.75$ & \\
\hline & $1,162.20$ & \\
\hline & $1,158.80$ & \\
\hline & $1,159.58$ & 1158.583 \\
\hline \multirow[t]{4}{*}{53} & $1,162.75$ & \\
\hline & $1,171.83$ & \\
\hline & $1,149.39$ & \\
\hline & $1,161.49$ & 1161.365 \\
\hline \multirow[t]{4}{*}{54} & $1,156.81$ & \\
\hline & $1,160.56$ & \\
\hline & $1,150.80$ & \\
\hline & $1,168.24$ & 1159.103 \\
\hline \multirow[t]{4}{*}{55} & $1,154.40$ & \\
\hline & $1,153.27$ & \\
\hline & $1,157.19$ & \\
\hline & $1,163.97$ & 1157.208 \\
\hline \multirow[t]{4}{*}{56} & $1,155.18$ & \\
\hline & $1,149.04$ & \\
\hline & $1,159.20$ & \\
\hline & $1,155.42$ & 1154.71 \\
\hline \multirow[t]{4}{*}{57} & $1,157.69$ & \\
\hline & $1,163.60$ & \\
\hline & $1,156.99$ & \\
\hline & $1,148.56$ & 1156.71 \\
\hline \multirow[t]{4}{*}{58} & $1,168.72$ & \\
\hline & $1,154.27$ & \\
\hline & $1,160.91$ & \\
\hline & $1,161.48$ & 1161.345 \\
\hline \multirow[t]{4}{*}{59} & $1,150.42$ & \\
\hline & $1,160.45$ & \\
\hline & $1,162.15$ & \\
\hline & $1,155.30$ & 1157.08 \\
\hline \multirow[t]{4}{*}{60} & $1,164.39$ & \\
\hline & $1,166.52$ & \\
\hline & $1,174.64$ & \\
\hline & $1,154.93$ & 1165.12 \\
\hline & 7.01 & 3.80 \\
\hline
\end{tabular}

Table S16. Signal Amplification - EtOH with 156 -170 ppm D noise evaluation

$$
\begin{array}{lr|rl}
\begin{array}{l}
\text { DET } \\
\text { NO. }
\end{array} & \multicolumn{1}{r}{\begin{aligned}
\text { READING } \\
\text { (PPM D) }
\end{aligned}} & \boldsymbol{\delta} \text { D (\%) } \\
\hline & \mathbf{1} & 174.33 & 131.3025 \\
& & &
\end{array}
$$




\begin{tabular}{|c|c|c|}
\hline 2 & 174.63 & 133.2497 \\
\hline 3 & 174.68 & 133.5742 \\
\hline 4 & 174.57 & 132.8602 \\
\hline 5 & 174.73 & 133.8987 \\
\hline 6 & 174.32 & 131.2376 \\
\hline 7 & 174.58 & 132.9251 \\
\hline 8 & 174.73 & 133.8987 \\
\hline 9 & 174.53 & 132.6006 \\
\hline 10 & 174.79 & 134.2882 \\
\hline 11 & 174.75 & 134.0285 \\
\hline 12 & 174.30 & 131.1078 \\
\hline 13 & 174.77 & 134.1584 \\
\hline 14 & 174.77 & 134.1584 \\
\hline 15 & 174.62 & 133.1848 \\
\hline 16 & 174.50 & 132.4059 \\
\hline 17 & 174.36 & 131.4972 \\
\hline 18 & 174.78 & 134.2233 \\
\hline 19 & 174.37 & 131.5621 \\
\hline 20 & 174.55 & 132.7304 \\
\hline 21 & 174.49 & 132.341 \\
\hline 22 & 174.43 & 131.9516 \\
\hline 23 & 174.62 & 133.1848 \\
\hline 24 & 174.49 & 132.341 \\
\hline 25 & 174.73 & 133.8987 \\
\hline 26 & 174.82 & 134.4829 \\
\hline 27 & 174.82 & 134.4829 \\
\hline 28 & 174.81 & 134.418 \\
\hline 29 & 174.56 & 132.7953 \\
\hline 30 & 174.72 & 133.8338 \\
\hline 31 & 174.22 & 130.5885 \\
\hline 32 & 174.88 & 134.8723 \\
\hline 33 & 174.65 & 133.3795 \\
\hline 34 & 174.80 & 134.3531 \\
\hline 35 & 174.47 & 132.2112 \\
\hline 36 & 174.16 & 130.1991 \\
\hline 37 & 174.13 & 130.0044 \\
\hline 38 & 174.80 & 134.3531 \\
\hline 39 & 174.29 & 131.0429 \\
\hline 40 & 174.54 & 132.6655 \\
\hline 41 & 174.42 & 131.8867 \\
\hline 42 & 174.81 & 134.418 \\
\hline 43 & 174.26 & 130.8482 \\
\hline 44 & 174.81 & 134.418 \\
\hline 45 & 174.58 & 132.9251 \\
\hline 46 & 174.61 & 133.1199 \\
\hline 47 & 174.54 & 132.6655 \\
\hline
\end{tabular}




\begin{tabular}{l|ll}
$\mathbf{4 8}$ & 174.82 & 134.4829 \\
$\mathbf{4 9}$ & 174.67 & 133.5093 \\
$\mathbf{5 0}$ & 175.14 & 136.5599 \\
$\mathbf{5 1}$ & 173.79 & 127.7976 \\
$\mathbf{5 2}$ & 174.39 & 131.6919 \\
$\mathbf{5 3}$ & 174.75 & 134.0285 \\
$\mathbf{5 4}$ & 174.51 & 132.4708 \\
$\mathbf{5 5}$ & 174.19 & 130.3938 \\
$\mathbf{5 6}$ & 174.97 & 135.4565 \\
$\mathbf{5 7}$ & 174.72 & 133.8338 \\
$\mathbf{5 8}$ & 174.52 & 132.5357 \\
$\mathbf{5 9}$ & 175.07 & 136.1055 \\
$\mathbf{6 0}$ & 174.95 & 135.3267 \\
& & \\
$\mathbf{5 D}$ & 0.24726 & 1.6049
\end{tabular}

Table S17. Signal Amplification - EtOH with 156 -170 ppm LOB/LOD

DET NO. CORRECTED $\delta \mathrm{D}(\%)$

\section{READING}

\begin{tabular}{l|ll}
\hline $\mathbf{1}$ & 155.5902 & -1.09052235 \\
& 155.4102 & -2.24632582 \\
\hline & 155.7202 & -0.25577515 \\
\hline $\mathbf{2}$ & 155.4902 & -1.73263544 \\
& 155.5302 & -1.47579022 \\
& 155.8702 & 0.707394973 \\
& 155.8502 & 0.578972274 \\
& 155.4202 & -2.18211453 \\
\hline $\mathbf{3}$ & 155.4602 & -1.92526934 \\
& 155.3802 & -2.43895969 \\
& 155.6302 & -0.83367708 \\
& 155.3202 & -2.82422739 \\
\hline $\mathbf{4}$ & 156.0002 & 1.542142646 \\
& 155.9702 & 1.349508548 \\
& 155.8502 & 0.578972274 \\
& 155.4702 & -1.86105804 \\
\hline $\mathbf{5}$ & 156.0302 & 1.734776755
\end{tabular}




\begin{tabular}{|c|c|c|}
\hline & 155.3502 & -2.63159355 \\
\hline & 155.3702 & -2.50317098 \\
\hline & 155.5802 & -1.15473367 \\
\hline \multirow[t]{4}{*}{6} & 155.8102 & 0.32212689 \\
\hline & 155.4602 & -1.92526934 \\
\hline & 156.3202 & 3.596907073 \\
\hline & 155.8602 & 0.643183623 \\
\hline \multirow[t]{4}{*}{7} & 155.6302 & -0.83367708 \\
\hline & 155.5202 & -1.54000153 \\
\hline & 155.8102 & 0.32212689 \\
\hline & 155.5802 & -1.15473367 \\
\hline \multirow[t]{4}{*}{8} & 155.5902 & -1.09052235 \\
\hline & 155.8702 & 0.707394973 \\
\hline & 156.0302 & 1.734776755 \\
\hline & 156.0602 & 1.927410876 \\
\hline \multirow[t]{4}{*}{9} & 155.4002 & -2.31053711 \\
\hline & 156.0402 & 1.798988128 \\
\hline & 155.6402 & -0.76946576 \\
\hline & 155.8702 & 0.707394973 \\
\hline \multirow[t]{4}{*}{10} & 156.0002 & 1.542142646 \\
\hline & 155.7102 & -0.31998648 \\
\hline & 155.5402 & -1.41157891 \\
\hline & 155.9002 & 0.900029032 \\
\hline \multirow[t]{4}{*}{11} & 156.1402 & 2.441101921 \\
\hline & 155.3802 & -2.43895969 \\
\hline & 155.5902 & -1.09052235 \\
\hline & 156.0002 & 1.542142646 \\
\hline \multirow[t]{4}{*}{12} & 155.6702 & -0.57683179 \\
\hline & 155.5202 & -1.54000153 \\
\hline & 155.9102 & 0.964240388 \\
\hline & 155.9602 & 1.285297185 \\
\hline \multirow[t]{4}{*}{13} & 155.7902 & 0.193704206 \\
\hline & 156.2802 & 3.340061448 \\
\hline & 156.0002 & 1.542142646 \\
\hline & 155.8502 & 0.578972274 \\
\hline \multirow[t]{4}{*}{14} & 156.0202 & 1.670565384 \\
\hline & 156.1102 & 2.24846777 \\
\hline & 155.9302 & 1.092663103 \\
\hline & 155.8602 & 0.643183623 \\
\hline \multirow[t]{4}{*}{15} & 156.1102 & 2.24846777 \\
\hline & 156.0202 & 1.670565384 \\
\hline & 155.7602 & 0.001070189 \\
\hline & 155.7602 & 0.001070189 \\
\hline SD & 0.254801 & 1.636113206 \\
\hline AVERAGE & 155.76 & 4.09999E-07 \\
\hline
\end{tabular}




$\begin{array}{lll} & \mathrm{ppm} & \delta \mathrm{D}(\%) \\ \text { LOB: } & 154.519 & 2.691407 \\ & & \\ \text { LOD: } & 154.9257 & 5.303273\end{array}$

\section{MATLAB Algorithm}

Direct copy of various MATLAB modules below with example values for testing parameters and .csv datafiles:

\section{Top Level Function:}

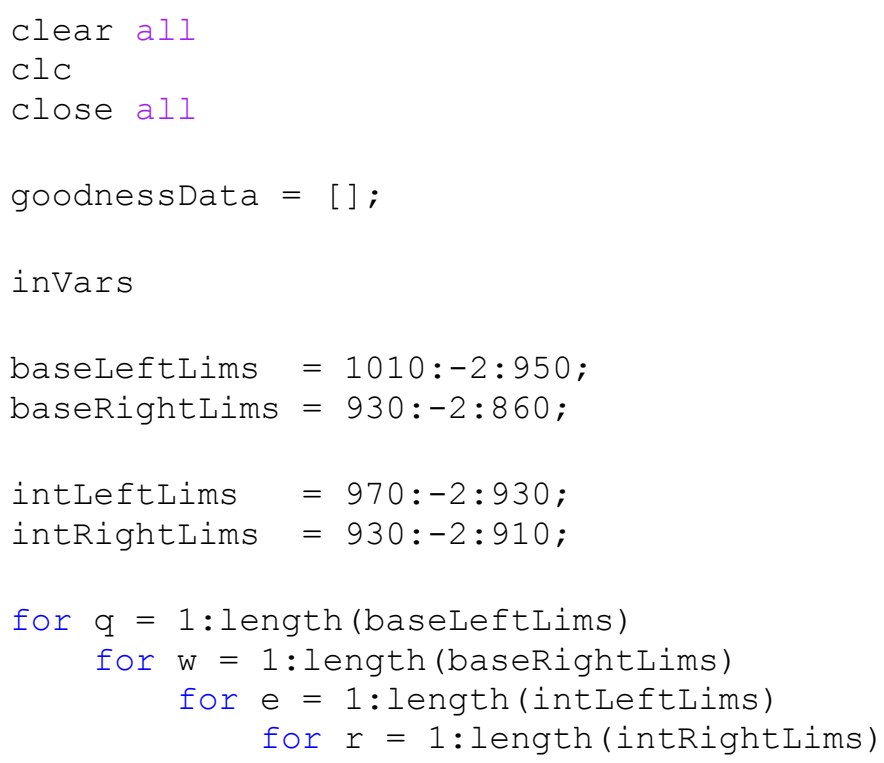




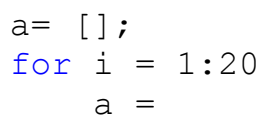

[a, dataIntegrator (baseLeftLims (q), baseRightLims (w), intLeftLims (e), intRightL ims (r), filenames (i,:)) ] ;

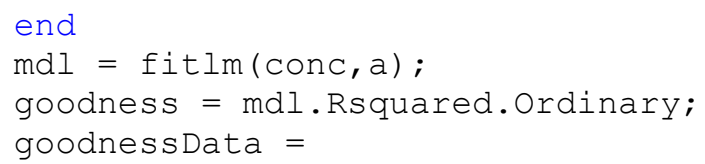

[goodnessData; goodness, baseLeftLims (q), baseRightLims (w), intLeftLims (e), intR ightLims ( $r)$ ] ; end end end

end

\section{inVars:}

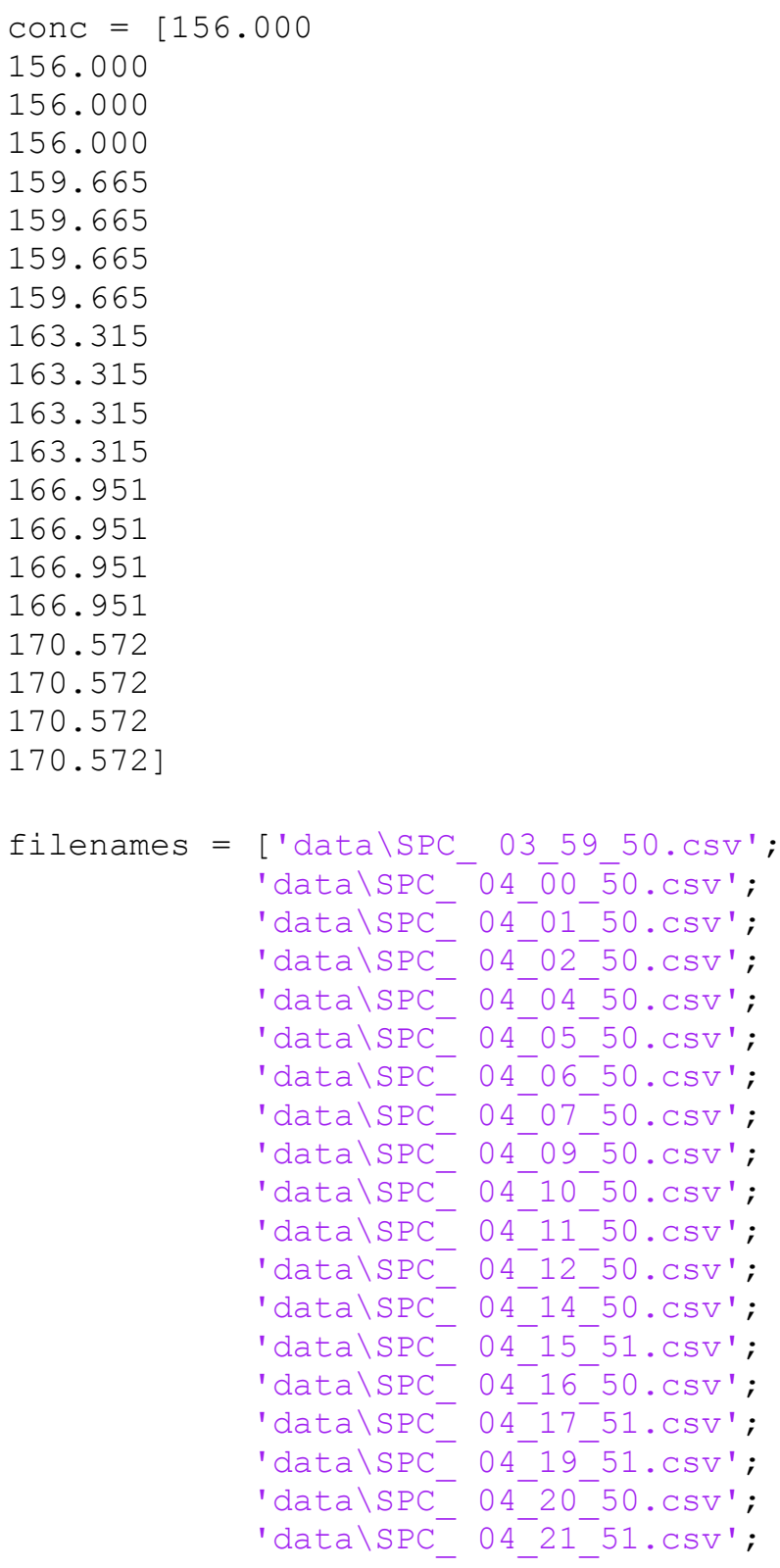


'data \SPC_04_22_50.CSV'];

\section{datalntegrator:}

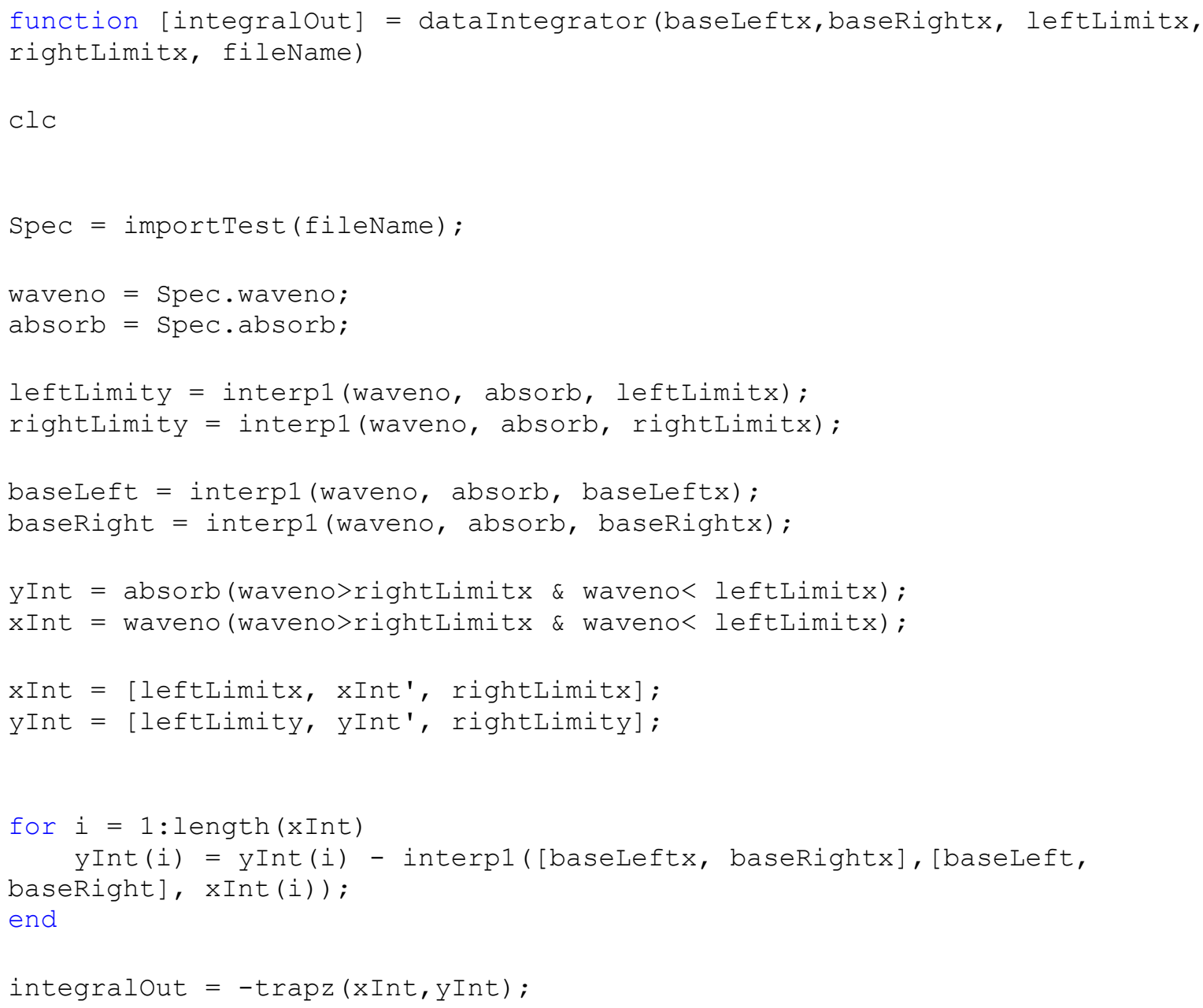

end

\section{importTest:}

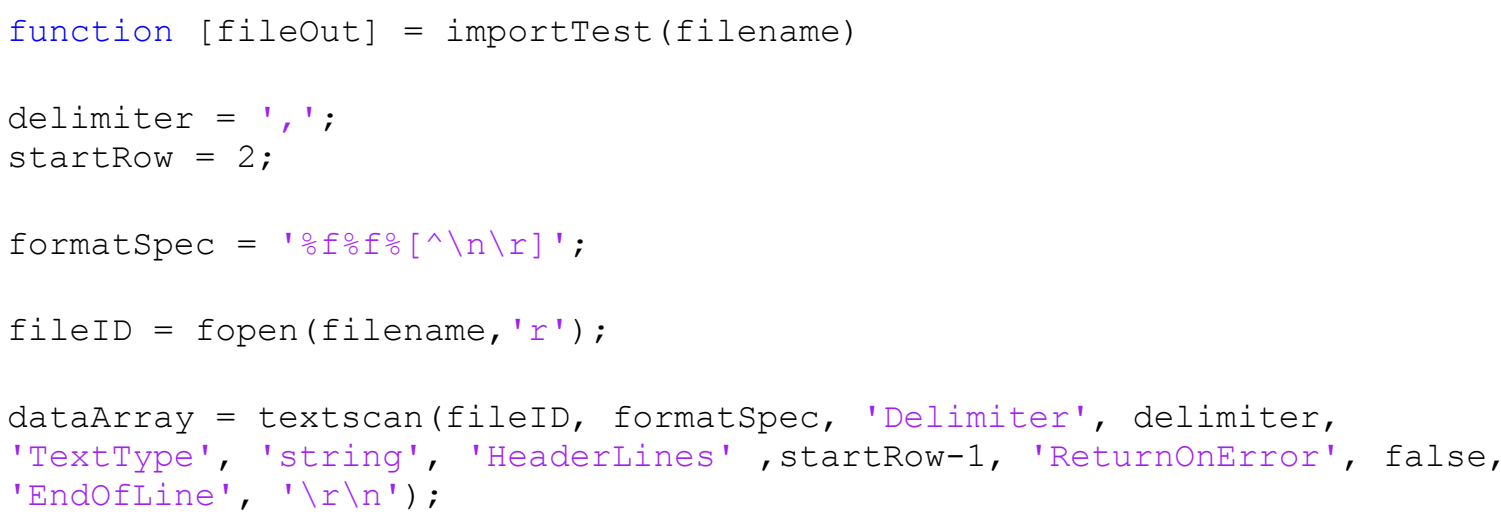




\section{Computational methods and additional considerations}

Optimisation and frequency calculations were carried out in order to generate simulated IR spectra for $\mathrm{H}_{2} \mathrm{O}, \mathrm{HDO}, \mathrm{D}_{2} \mathrm{O}, \mathrm{HTO}$, DTO, $\mathrm{T}_{2} \mathrm{O}$, and for all combination of $\mathrm{ROX}+\mathrm{X}_{2} \mathrm{O}(\mathrm{R}=\mathrm{Me}, \mathrm{Et}$, and $X=H, D, T)$. All structures were optimised by DFT calculations carried out using Gaussian16 (Revision A.03) ${ }^{6}$ with the B3LYP density functional, ${ }^{7,8}$ dispersion-corrected with the D3 version of Grimme's dispersion ${ }^{9}$ with Becke-Johnson damping (D3(BJ)). ${ }^{10-12}$ The polarised triple- $\zeta$ valence quality (def2-TZVPP) basis $\operatorname{set}^{13}$ and the integral equation formalism version of the polarisable continuum model (IEF-PCM(water)) $)^{14}$ were also used. All calculations were performed with an ultrafine integration grid. Temperature (298.15K) and concentration-corrected (1 mol/l) quasiharmonic (Grimme approximation ${ }^{15}$ ) free energies were calculated with GoodVibes. ${ }^{16}$

Because proton exchange is not accounted for by the DFT calculations, natural broadening of peaks does not occur in the simulated spectra. However, the relevant positions of peaks were found to be sufficiently accurate such that the quantitative data and trends provided by the simulated spectra could be reliably interpreted. Furthermore, because methanol and ethanol were only modelled with one explicit molecule of water present, all of the species that would exist in such a solution, for example as a result of proton exchange with deuterated or tritiated water, were not represented simultaneously in the calculations. However, key IR peaks resulting from each species individually could still be identified. 
To assess the influence of hydrogen-bonding on the simulated spectra, additional calculations were carried out for all combinations of the ROX $+3 \mathrm{X}_{2} \mathrm{O}$ system (Figure S3), however this resulted in more complex spectra that illustrate the same general trends. A similar result was found upon inclusion of anharmonicity (Figure S4), and thus all spectra presented in the main text employ only the double harmonic approximation. These additional spectra are presented below, with absorbance scaled by a factor of 0.333 in all cases relative to the harmonic spectra in the manuscript.

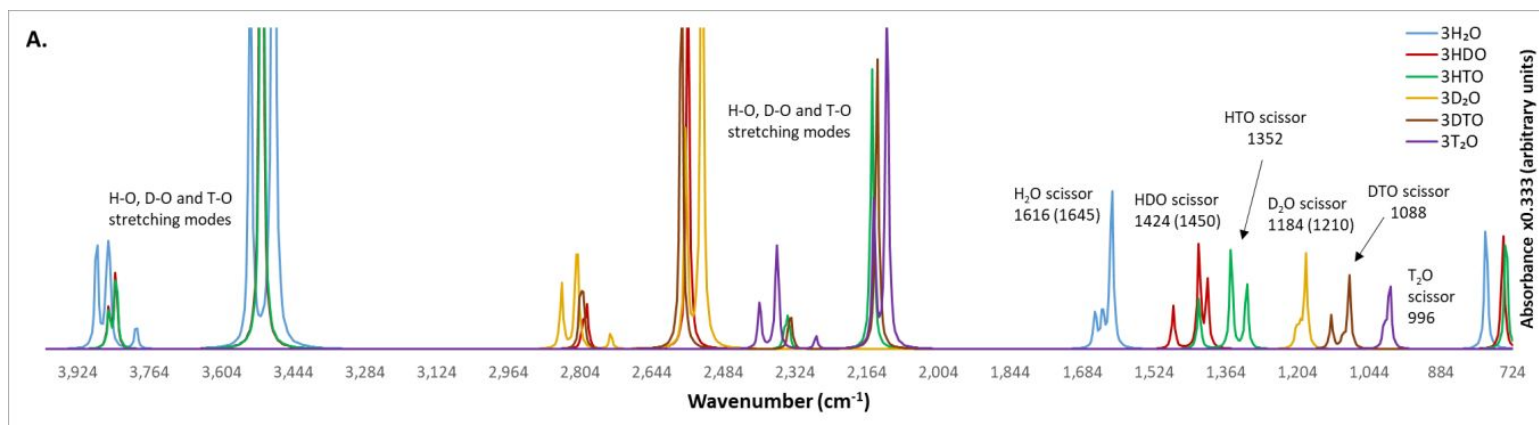

B.
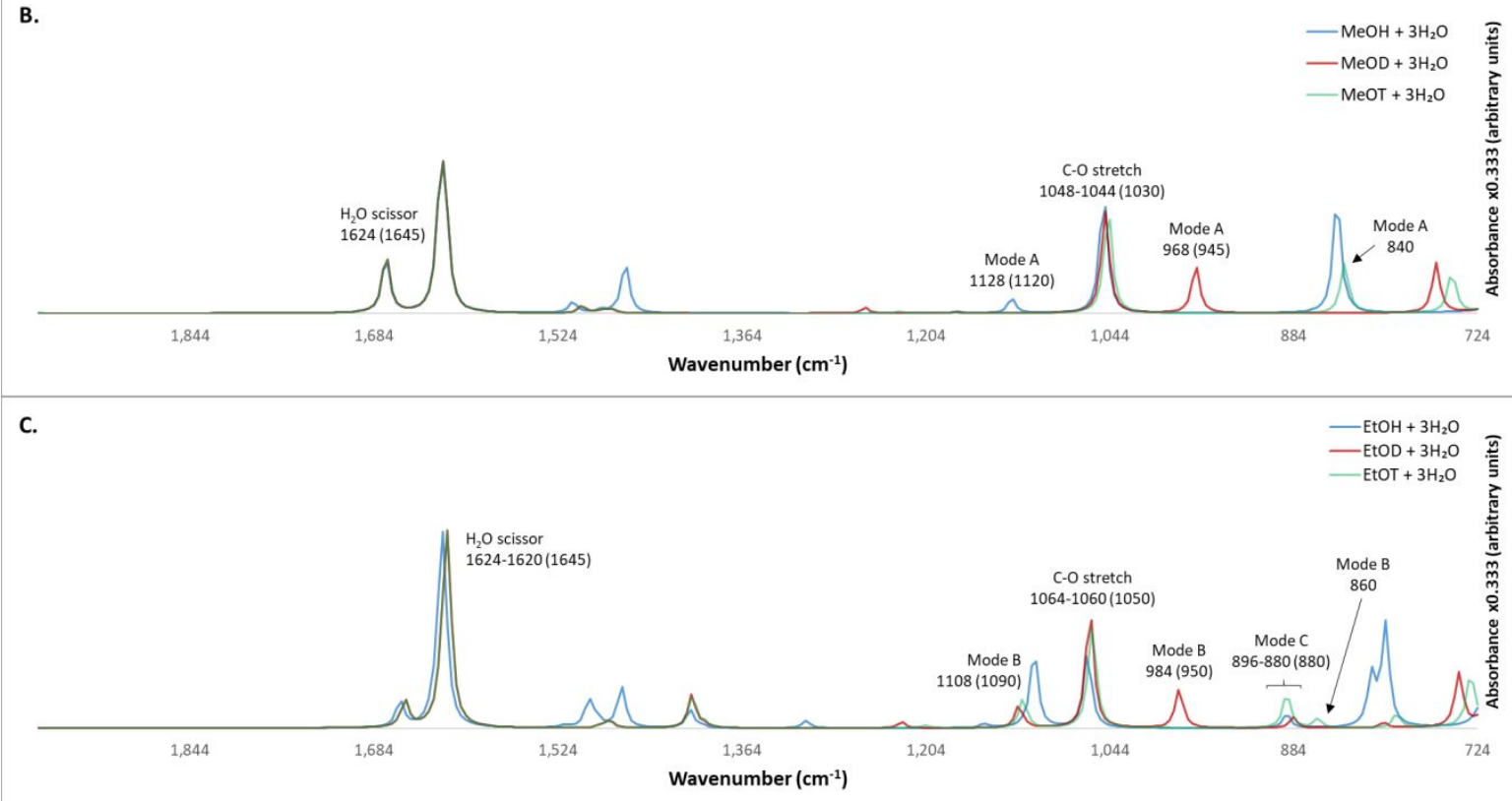

D.

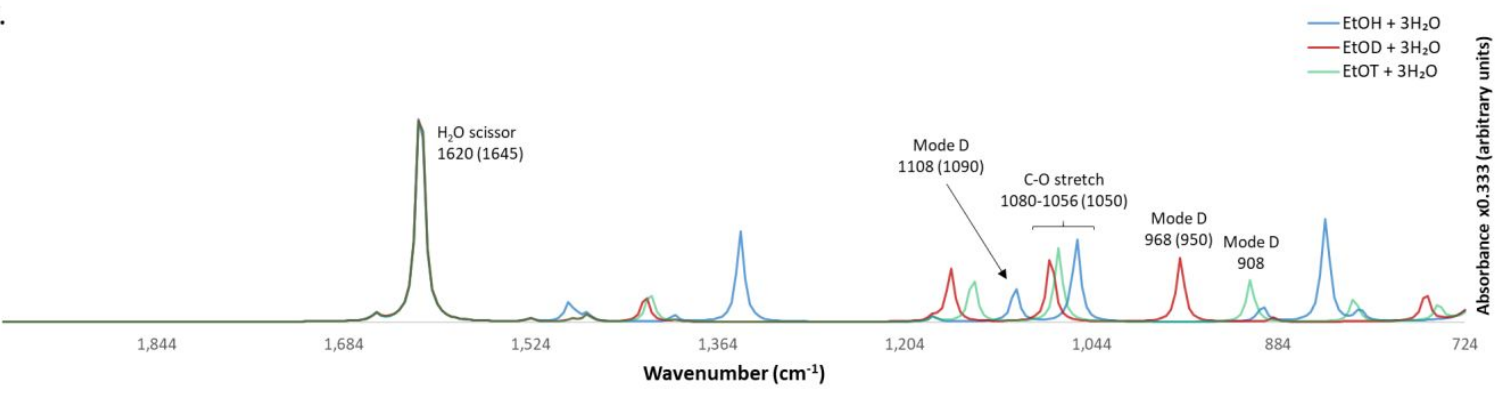

Figure S3: Simulated IR spectrum for all (A) isotopologues of three molecules of water; (B) isotopologues of methanol with three water molecules; $(C)$ isotopologues of gauche-ethanol with three water molecules; (D) isotopologues of anti-ethanol with three water molecules. Experimental frequencies are provided in brackets where available. 


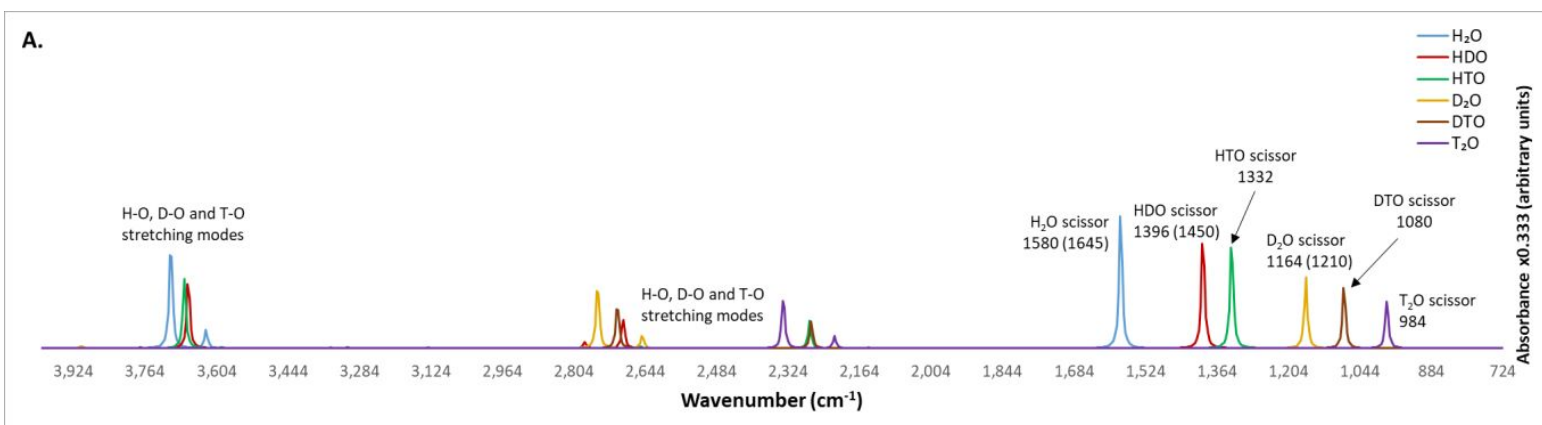

B.
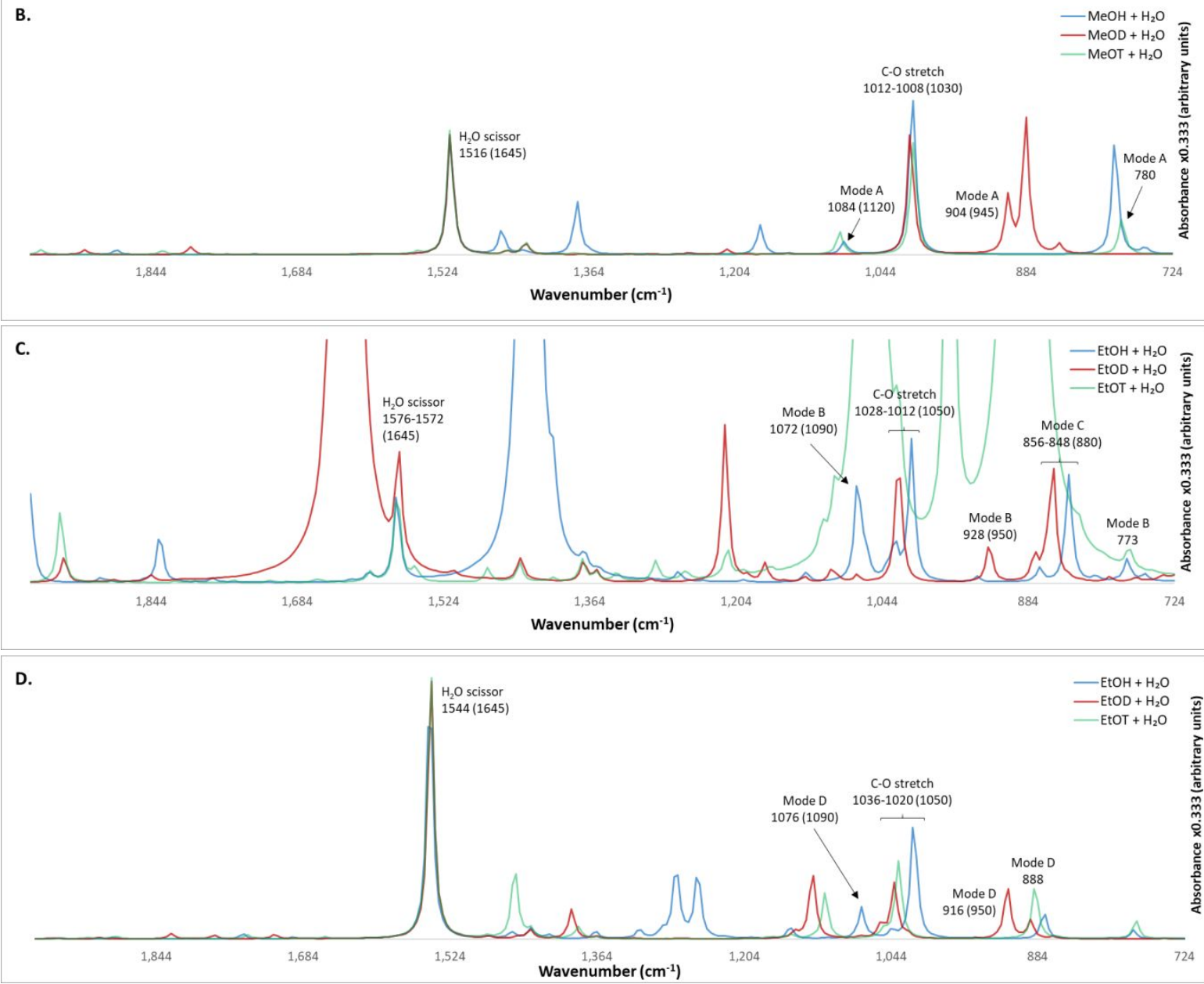

Figure S4: Simulated anharmonic IR spectrum for all (A) isotopologues of water; (B) isotopologues methanol with water; (C) isotopologues of gauche-ethanol with water; (D) isotopologues of anti-ethanol with water. Experimental frequencies are provided in brackets where available. 


\section{Alternative signal amplifiers}

Some further work was carried out in order to identify a more effective signal amplifying molecule than ethanol. Identification of such a molecule could potentially lead to a significant increase in the sensitivity of the quantification technique. Potential signal amplifying molecules should possess an exchangeable proton, and should produce a clean spectrum with one or more clearly resolvable and sufficiently intense isotope-sensitive peak(s) in the deuterated and tritiated spectrum. As the experimental testing of many different molecules is impractical, time-consuming, and expensive, potential candidates were instead identified by simulation of their IR spectra by DFT and subsequent comparison to their corresponding NIST experimental spectra (where available). The most promising candidates were tested experimentally in water and D2O to assess their overall potential as signal amplifying molecules.

A number of potential classes of alcoholic signal amplifiers were considered. Linear alcohols up to 12 carbons were found to become less suitable candidates as the number of carbons was increased, causing peaks in the region of interest to become less intense and more complex. The IR spectra of linear diols were similar in appearance to their analogous linear alcohols, but slightly more complex, and thus less effective as signal amplifiers. Fatty alcohols up to 18 carbons were found to possess complicated IR spectra with relatively small peaks, thus limiting the potential resolution and sensitivity of the quantification technique through their use. Although the IR spectra of some small saturated alcohols, such as ethenol and ethynol, appeared promising, each of these species exists in another significantly more stable tautomeric form, presenting severe practical issues. The IR spectra of some conformationally simple alcohols were also promising, namely that for tert-Butanol, which exhibited three separate isotope-sensitive peaks in promising regions of the spectra in both the computed and ReactIR spectra (Figure S5). However, whilst experimental testing of tert-Butanol indicated that a sensitivity at least as good as with ethanol could potentially be achieved, a number of practical issues relating to the melting point and viscosity of the chemical were identified. Computational modelling of some other branched alcohols resulted in more complex IR spectra than those for ethanol.
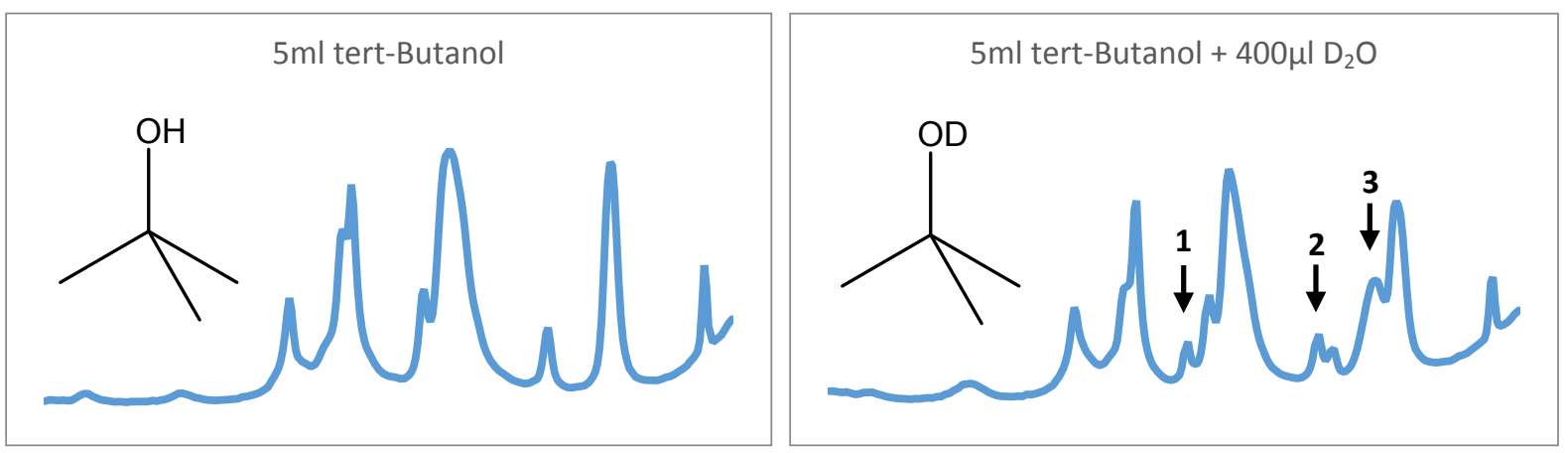

Figure S5: Experimental (ReactIR) spectra for tert-butanol. Spectra within ReactIR range $\left(1900-700 \mathrm{~cm}^{-1}\right)$. Arrows indicate isotope-sensitive peaks. 


\section{Energies and molecular geometries of all computed structures}

All energies in Hartrees, coordinates in $\AA$.

\section{Water:}

B3LYP-D3(BJ)/def2-TZVPP-IEF-PCM(water) Energy $=-76.474050$

B3LYP-D3(BJ)/def2-TZVPP-IEF-PCM(water) Free Energy (Quasiharmonic) $=-76.467458$

B3LYP-D3(BJ)/def2-TZVPP-IEF-PCM(water) Molecular Geometry in Cartesian Coordinates:

$\begin{array}{llll}\mathrm{O} & 0.000000 & 0.000000 & 0.117919 \\ \mathrm{H} & 0.000000 & 0.760106 & -0.471676 \\ \mathrm{H} & -0.000000 & -0.760106 & -0.471676\end{array}$

\section{Water (3):}

B3LYP-D3(BJ)/def2-TZVPP-IEF-PCM(water) Energy = -229.439202

B3LYP-D3(BJ)/def2-TZVPP-IEF-PCM(water) Free Energy (Quasiharmonic) $=-229.397147$

B3LYP-D3(BJ)/def2-TZVPP-IEF-PCM(water) Molecular Geometry in Cartesian Coordinates:

$\begin{array}{llll}\mathrm{O} & -2.331621 & -0.590096 & 0.094152 \\ \mathrm{H} & -1.548386 & -0.008684 & 0.039150 \\ \mathrm{H} & -2.774535 & -0.487012 & -0.752320 \\ \mathrm{O} & 2.195607 & -0.537392 & -0.080507 \\ \mathrm{H} & 3.026532 & -0.058363 & -0.165667 \\ \mathrm{H} & 2.292488 & -1.069043 & 0.716461 \\ \mathrm{O} & -0.079420 & 1.055798 & -0.071064 \\ \mathrm{H} & -0.014970 & 1.670277 & 0.665737 \\ \mathrm{H} & 0.742348 & 0.526348 & -0.044008\end{array}$

\section{Methanol + water:}

B3LYP-D3(BJ)/def2-TZVPP-IEF-PCM(water) Energy = -192.268922

B3LYP-D3(BJ)/def2-TZVPP-IEF-PCM(water) Free Energy (Quasiharmonic) $=-192.220063$

B3LYP-D3(BJ)/def2-TZVPP-IEF-PCM(water) Molecular Geometry in Cartesian Coordinates:

$\begin{array}{llll}\mathrm{O} & 0.734078 & -0.736342 & 0.013015 \\ \mathrm{H} & -0.196278 & -0.455163 & 0.006184 \\ \mathrm{O} & -1.924459 & 0.210039 & -0.012909 \\ \mathrm{H} & -2.411647 & 0.027907 & 0.797248\end{array}$

S-60 


$\begin{array}{llll}\mathrm{C} & 1.544176 & 0.430457 & -0.007659 \\ \mathrm{H} & 1.372383 & 1.064810 & 0.867901 \\ \mathrm{H} & 2.586031 & 0.112315 & 0.000718 \\ \mathrm{H} & 1.376147 & 1.030731 & -0.907627 \\ \mathrm{H} & -2.468643 & -0.152916 & -0.719324\end{array}$

\section{Methanol + water (3):}

B3LYP-D3(BJ)/def2-TZVPP-IEF-PCM(water) Energy = -345.235283

B3LYP-D3(BJ)/def2-TZVPP-IEF-PCM(water) Free Energy (Quasiharmonic) $=-345.146230$

B3LYP-D3(BJ)/def2-TZVPP-IEF-PCM(water) Molecular Geometry in Cartesian Coordinates:

$\begin{array}{lrrr}\mathrm{O} & 1.691401 & -1.037889 & 0.667677 \\ \mathrm{H} & 0.866799 & -0.571590 & 0.911531 \\ \mathrm{O} & -0.667036 & 0.327908 & 1.149266 \\ \mathrm{H} & -0.503576 & 1.142926 & 0.640776 \\ \mathrm{C} & 1.835759 & -0.932820 & -0.740045 \\ \mathrm{H} & 1.915238 & 0.109298 & -1.067772 \\ \mathrm{H} & 2.750959 & -1.450942 & -1.025052 \\ \mathrm{H} & 0.999307 & -1.396081 & -1.274417 \\ \mathrm{H} & -1.311475 & -0.182929 & 0.626184 \\ \mathrm{O} & -0.053019 & 2.651421 & -0.337757 \\ \mathrm{H} & 0.862934 & 2.905555 & -0.183232 \\ \mathrm{H} & -0.105657 & 2.479605 & -1.283811 \\ \mathrm{O} & -2.438442 & -1.263804 & -0.361972 \\ \mathrm{H} & -2.783903 & -0.824746 & -1.146211 \\ \mathrm{H} & -1.968411 & -2.035263 & -0.695447\end{array}$

\section{Anti-ethanol + water:}

B3LYP-D3(BJ)/def2-TZVPP-IEF-PCM(water) Energy = -231.607044

B3LYP-D3(BJ)/def2-TZVPP-IEF-PCM(water) Free Energy (Quasiharmonic) $=-231.531686$

B3LYP-D3(BJ)/def2-TZVPP-IEF-PCM(water) Molecular Geometry in Cartesian Coordinates:

$\begin{array}{llll}\mathrm{O} & 0.025898 & -0.717266 & 0.011281 \\ \mathrm{H} & -0.903651 & -0.433295 & 0.004850 \\ \mathrm{O} & -2.647679 & 0.207389 & -0.011706 \\ \mathrm{H} & -3.128731 & 0.009424 & 0.798420 \\ \mathrm{C} & 0.848554 & 0.446729 & -0.009958 \\ \mathrm{H} & 0.636880 & 1.078901 & 0.860157\end{array}$




$\begin{array}{cccc}\mathrm{H} & 0.640678 & 1.043991 & -0.905280 \\ \mathrm{H} & -3.183468 & -0.167201 & -0.718490 \\ \mathrm{C} & 2.301662 & 0.020845 & 0.001641 \\ \mathrm{H} & 2.955031 & 0.894103 & -0.014618 \\ \mathrm{H} & 2.526309 & -0.558359 & 0.898496 \\ \mathrm{H} & 2.529910 & -0.593995 & -0.870237\end{array}$

\section{Anti-ethanol + water (3):}

B3LYP-D3(BJ)/def2-TZVPP-IEF-PCM(water) Energy = -384.572711

B3LYP-D3(BJ)/def2-TZVPP-IEF-PCM(water) Free Energy (Quasiharmonic) $=-384.458842$

B3LYP-D3(BJ)/def2-TZVPP-IEF-PCM(water) Molecular Geometry in Cartesian Coordinates:

$\begin{array}{llll}\mathrm{O} & 1.367187 & -0.017881 & -0.594236 \\ \mathrm{H} & 0.479404 & -0.015065 & -0.182172 \\ \mathrm{O} & -1.099422 & -0.006072 & 0.638559 \\ \mathrm{H} & -1.631621 & -0.775412 & 0.366797 \\ \mathrm{C} & 2.338374 & -0.010056 & 0.445681 \\ \mathrm{H} & 2.215356 & -0.888308 & 1.090948 \\ \mathrm{H} & 2.213736 & 0.876529 & 1.079128 \\ \mathrm{H} & -1.611858 & 0.780085 & 0.377136 \\ \mathrm{C} & 3.723500 & -0.012885 & -0.167803 \\ \mathrm{H} & 4.487130 & -0.007101 & 0.611215 \\ \mathrm{H} & 3.870063 & -0.901238 & -0.784157 \\ \mathrm{H} & 3.868526 & 0.867537 & -0.795785 \\ \mathrm{O} & -2.550407 & -2.288334 & -0.146727 \\ \mathrm{O} & -2.486966 & 2.324642 & -0.119465 \\ \mathrm{H} & -3.401955 & 2.360942 & 0.177958 \\ \mathrm{H} & -2.531504 & 2.423053 & -1.076194 \\ \mathrm{H} & -2.789007 & -2.273752 & -1.079361 \\ \mathrm{H} & -3.382647 & -2.408458 & 0.322162\end{array}$

\section{Gauche-ethanol + water:}

B3LYP-D3(BJ)/def2-TZVPP-IEF-PCM(water) Energy = -231.607298

B3LYP-D3(BJ)/def2-TZVPP-IEF-PCM(water) Free Energy (Quasiharmonic) $=-231.531569$

B3LYP-D3(BJ)/def2-TZVPP-IEF-PCM(water) Molecular Geometry in Cartesian Coordinates:
$\begin{array}{llll}O & -0.156947 & -1.107719 & -0.354667\end{array}$
H $\quad 0.673787 \quad-0.620041 \quad-0.222135$ 


$\begin{array}{lrrr}\mathrm{O} & 2.234271 & 0.339373 & 0.130949 \\ \mathrm{H} & 2.566293 & 0.813237 & -0.638590 \\ \mathrm{C} & -1.188460 & -0.423648 & 0.351992 \\ \mathrm{H} & -0.980178 & -0.429046 & 1.428450 \\ \mathrm{H} & -2.099422 & -1.003446 & 0.196407 \\ \mathrm{H} & 2.969740 & -0.208511 & 0.423952 \\ \mathrm{C} & -1.385173 & 1.005405 & -0.127917 \\ \mathrm{H} & -2.202160 & 1.483203 & 0.416423 \\ \mathrm{H} & -0.481341 & 1.595372 & 0.033183 \\ \mathrm{H} & -1.623516 & 1.025458 & -1.192395\end{array}$

\section{Gauche-ethanol + water (3):}

B3LYP-D3(BJ)/def2-TZVPP-IEF-PCM(water) Energy = -384.573462

B3LYP-D3(BJ)/def2-TZVPP-IEF-PCM(water) Free Energy (Quasiharmonic) $=\mathbf{- 3 8 4 . 4 5 7 7 3 4}$

B3LYP-D3(BJ)/def2-TZVPP-IEF-PCM(water) Molecular Geometry in Cartesian Coordinates:

$\begin{array}{llll}\mathrm{O} & 1.117618 & -1.424686 & 0.518584 \\ \mathrm{H} & 0.368034 & -0.946003 & 0.109934 \\ \mathrm{O} & -0.970092 & -0.061290 & -0.690411 \\ \mathrm{H} & -0.918555 & 0.861414 & -0.383517 \\ \mathrm{C} & 2.312822 & -1.026425 & -0.142387 \\ \mathrm{H} & 2.282168 & -1.320817 & -1.198777 \\ \mathrm{H} & 3.124362 & -1.587926 & 0.323815 \\ \mathrm{H} & -1.845905 & -0.387975 & -0.416207 \\ \mathrm{C} & 2.582311 & 0.467045 & -0.035604 \\ \mathrm{H} & 3.529815 & 0.720570 & -0.515468 \\ \mathrm{H} & 1.793080 & 1.040983 & -0.523224 \\ \mathrm{H} & 2.633425 & 0.775214 & 1.009946 \\ \mathrm{O} & -0.694665 & 2.591210 & 0.234923 \\ \mathrm{H} & -0.349611 & 3.194682 & -0.431338 \\ \mathrm{H} & -0.051277 & 2.617165 & 0.950857 \\ \mathrm{O} & -3.466734 & -1.097022 & 0.108000 \\ \mathrm{H} & -4.223491 & -0.650427 & -0.285546 \\ \mathrm{H} & -3.601848 & -1.026291 & 1.058704\end{array}$




\section{References}

(1) ReactIR $15 \quad$ - $\quad$ Overview https://www.mt.com/gb/en/home/products/L1_AutochemProducts/ReactIR/ReactIR -15.html (accessed Dec 10, 2018).

(2) Rohman, A.; Musfiroh, A.; Wijaya, E. G. Quantitative Determination of Simethicone in Antacid Suspension and Chewable Tablet Using FTIR Spectroscopy. Glob. J. Pharmacol. 2013, 7 (3), 270-275.

(3) Armbruster, D. A.; Pry, T. Limit of Blank, Limit of Detection and Limit of Quantitation. Clin. Biochem. Rev. 2008, 29 Suppl 1 (August), S49-52. https://doi.org/citeulike-articleid:3416410.

(4) International Conference on Harmonization (ICH). Guidance for Industry: Q2B Validation of Analytical Procedures: Methodology. 1996.

(5) Shabir, G. Step-by-Step Analytical Methods Validation and Protocol in the Quality System Compliance Industry. J. Valid. Technol. 2004, 10, 210-218.

(6) Frisch, M. J.; Trucks, G. W.; Schlegel, H. B.; Scuseria, G. E.; Robb, M. A.; Cheeseman, J. R.; Scalmani, G.; Barone, V.; Mennucci, B.; Petersson, G. A.; Nakatsuji, H.; Caricato, M.; Li, X.; Hratchian, H. P.; Izmaylov, A. F.; Bloino, J.; Zheng, J.; Sonnenberg, J. L.; Hada, M.; Ehara, M.; Toyota, K.; Fukuda, R.; Hasegawa, J.; Ishida, M.; Nakajima, T.; Honda, Y.; Kitao, O.; Nakai, H.; Vreven, T.; Montgomery, J. A.; Peralta, J. E.; Ogliaro, F.; Bearpark, M.; Heyd, J. J.; Brothers, E.; Kudin, K. N.; Staroverov, V. N.; Kobayashi, R.; Normand, J.; Raghavachari, K.; A. Rendell, J. C.; Burant, S.; lyengar, S.; Tomasi, J.; Cossi, M.; Rega, N.; Millam, J. M.; Klene, M.; Knox, J. E.; Cross, J. B.; Bakken, V.; Adamo, C.; Jaramillo, J.; Gomperts, R.; Stratmann, R. E.; Yazyev, O.; Austin, A. J.; Cammi, R.; Pomelli, C.; Ochterski, J. W.; Martin, R. L.; Morokuma, K.; Zakrzewski, V. G.; Voth, G. A.; Salvador, P.; Dannenberg, J. J.; Dapprich, S.; Daniels, A. D.; Farkas, O.; Foresman, J. B.; Ortiz, J. V.; Cioslowski, J.; Fox, D. J. Gaussian 16, Revision A.03. Gaussian, Inc.: Wallingford CT 2016.

(7) Becke, A. D. Density-functional Thermochemistry. III. The Role of Exact Exchange. J. Chem. Phys. 1993, 98 (7), 5648-5652.

(8) Stephens, P. J.; Devlin, F. J.; Chabalowski, C. F.; Frisch, M. J. Ab Initio Calculation of Vibrational Absorption and Circular Dichroism Spectra Using Density Functional Force Fields. J. Phys. Chem. 1994, 98 (45), 11623-11627.

(9) Grimme, S.; Antony, J.; Ehrlich, S.; Krieg, H. A Consistent and Accurate Ab Initio Parametrization of Density Functional Dispersion Correction (DFT-D) for the 94 Elements H-Pu. J. Chem. Phys. 2010, 132 (15), 154104.

(10) Becke, A. D.; Johnson, E. R. A Density-Functional Model of the Dispersion Interaction. J. Chem. Phys. 2005, 123 (15), 154101.

(11) Johnson, E. R.; Becke, A. D. A Post-Hartree-Fock Model of Intermolecular Interactions: Inclusion of Higher-Order Corrections. J. Chem. Phys. 2006, 124 (17), 174104.

(12) Grimme, S.; Ehrlich, S.; Goerigk, L. Effect of the Damping Function in Dispersion Corrected Density Functional Theory. J. Comput. Chem. 2011, 32 (7), 1456-1465. 
(13) Weigend, F.; Ahlrichs, R. Balanced Basis Sets of Split Valence, Triple Zeta Valence and Quadruple Zeta Valence Quality for $\mathrm{H}$ to Rn: Design and Assessment of Accuracy. Phys. Chem. Chem. Phys. 2005, 7 (18), 3297.

(14) Mennucci, B.; Cammi, R.; Tomasi, J. Excited States and Solvatochromic Shifts within a Nonequilibrium Solvation Approach: A New Formulation of the Integral Equation Formalism Method at the Self-Consistent Field, Configuration Interaction, and Multiconfiguration Self-Consistent Field Level. J. Chem. Phys. 1998, 109 (7), 2798-2807.

(15) Grimme, S. Supramolecular Binding Thermodynamics by Dispersion-Corrected Density. Chem. Eur. J. 2012, 18 (32), 9955-9964.

(16) Funes-Ardoiz, I.; Paton, R. S. Goodvibes: Version 2.0.3. 2018. 